\title{
Zeitschriftenlese
}

AfP

Jg 36 (2005) Nr 2

Lorz, Ralph Alexander: Rechtliche Parameter für die Öffentlichkeitsarbeit der Justiz: eine aktuelle Analyse aus Anlass des sog. „Mannesmann“-Verfahrens. - S. 97-109

„Die Klage das ehemaligen Vorstandsvorsitzenden der Mannesmann-AG, Dr. Klaus Esser, gegen Verlautbarungen der Düsseldorfer Staatsanwaltschaft im Zusammenhang mit einem Strafverfahren gehen ihn hat zu erheblicher Resonanz in der Öffentlichkeit geführt. Der vorliegende Beitrag befasst sich nach der inzwischen ergangenen Entscheidung des LG Düsseldorf mit der Frage, welche rechtlichen Parameter für die Öffentlichkeitsarbeit der Justiz zu gelten haben. Dabei wird der Beitrag zunächst die zu beachtenden Rechtsgüter darstellen, um im Anschluss hieran die Überlegungen des Landgerichtes Düsseldorf kritisch zu beleuchten."

Born, Christian: Gen-Milch und Goodwill: Äußerungsrechtlicher Schutz durch das Unternehmenspersönlichkeitsrecht. - S. 110-117

Bekannte Marken sind längst nicht mehr nur Individualisierungszeichen eines Unternehmens. Über die ursprüngliche Herkunftsfunktion hinaus stellt die Marke nicht selten den wertvollsten Vermögensgegenstand des Unternehmens dar. Das regelmäßig unter hohem Aufwand erarbeitete „gute Image“ des Unternehmens und seiner Marken will daher auch bestmöglich gegen Diffamierungen und Maßnahmen öffentlicher Kritik verteidigt werden. Gegenstand des vorliegenden Beitrages ist in diesem Zusammenhang mit Blick auf die "Gen-Milch"-Kampagne von Greenpeace - das von der Rechtsprechung entwickelte Unternehmenspersönlichkeitsrecht, wobei insbesondere auf die Frage der grundsätzlichen Notwendigkeit der Gewährung einer Geldentschädigung, also der Kompensation immaterieller Schäden in Geld im Fall der Verletzung des Unternehmenspersönlichkeitsrechtes eingegangen wird.

Kloepfer, Michael: Datenschutz in Redaktionen: Bilanz und Perspektiven. - S. 118-126

Der Datenschutz in Massenmedien ist in Deutschland unterschiedlich nach den jeweiligen Medien geregelt. Der hier näher interessierende Redaktionsdatenschutz bei der Presse steht seit der Überarbeitung des datenschutzrechtlichen Medienprivilegs des $\$ 41$ I Bundesdatenschutzgesetz (BDSG) im Jahre 2001 auf zwei Säulen: einer normativen, rechtlich verbindlichen staatlichen Säule und einer Säule der sog. freiwilligen Selbstkontrolle durch die Presse selbst. Die normative Säule besteht hierbei aus dem BDSG und den Pressegesetzen der Länder, während sich die Säule der freiwilligen Selbstkontrolle aus vielen einzelnen Selbstverpflichtungserklärungen von Verlagsunternehmen zusammensetzt, welche auf die quasi standesrechtlichen Regelwerke des Deutschen Presserates Bezug nehmen. Auf den Inhalt dieser beiden Säulen wird im vorliegendem Beitrag eingegangen.
Dreher, Meinrad: Die vergaberechtliche Beurteilung von Aufträgen zur Ausstrahlung von Rundfunksendungen. - S. 127-132

Beater, Axel: Bildinformation im Medienrecht. -S. 133-139

Das Recht unterscheidet an vielen Stellen zwischen Wort und Bild. Das Äußerungsdeliktsrecht etwa kennt die Unterscheidung zwischen Wort- und Bildberichterstattung, das Urheberrecht die zwischen Sprach-, Lichtbild- und Filmwerk. Ebenso gibt es zahlreiche Einzelregelungen, die zwischen Sprache und Bild trennen und jeweils für eines von beiden besondere Regeln aufstellen. Vor diesem Hintergrund beschäftigt sich vorliegender Beitrag mit den Besonderheiten von Bildern, d. h. deren Wirkung und Bedeutung für die Medien. Hierbei wird insbesondere auf ihre Informations-, Dokumentations-, Auseinandersetzungs- und Illustrationsfunktion sowie auf das kommerzielle Nutzungsinteresse eingegangen.

Weberling, Johannes: Unterlassungsansprüche des Betriebsrats bei Verstößen gegen $\$ 90$ BetrVG insbesondere in Tendenzunternehmen. - S. 139-141

Groß, Rolf: Öffentliche Aufgabe und Verantwortlichkeit der Presse. - S. 142-151

Massenmedien oder Massenkommunikationsmittel wie die Presse erfüllen im demokratischen Staat die Aufgabe, das Staatsvolk zu unterrichten und in die Lage zu versetzen, die staatsbürgerlichen Rechte auszuüben. Ohne ihre Vermittlung kann sich der Staatsbürger im modernen Flächenstaat kein Bild über die Ausübung der Staatsgewalt machen. Transparenz der Herrschaftsausübung ist zwar Voraussetzung für die Information des Bürgers, gewährleistet sie aber nicht, solange die Informationsvermittlung fehlt. Vor diesem Hintergrund beschäftigt sich vorliegender Beitrag mit der öffentlichen Aufgabe, der Wahrhaftigkeit und Sorgfalt der Presse, sowie auf der anderen Seite mit Rechtsgütern Dritter und deren Ansprüche gegen die Presse.

\section{Communication Theory}

\section{Jg 15 (2005) Nr 2}

Knight Lapinski, Maria: An Explication of Social Normes. - S. 127-147

Mayer, Vicki: Research Beyond the Pale: Whiteness in Audience Studies and Media Ethnography. - S. 148-167

Tracy, Sarah J.: Fracturing the RealSelf $<>$ Fake-Self Dichotomy: Moving Toward „Crystallized“ Organizational Discourses and Identities. - S. 168-195

Juluri, Vamsee: Nonviolence and Media Studies. - S. 196-215 


\section{Communications}

\section{Jg 30 (2005) Nr 2}

Hetsroni, Amir: Rule Britannia!; Britannia rules the waves: A cross-cultural study of five English-speaking versions of a British quiz show format. - S. 129-153

"This study analyzed the content of questions in the quiz show Who Wants to be a Millionaire in the UK, the USA, Australia, Singapore, and India. A total of 1,823 questions were sampled (427 from British programs, 374 from American programs, 324 from Australian programs, 349 from Singapore, and 349 from India). The topics of questions varied from country to country with programs in Australia and the UK overrepresenting language, the USA and Singapore overrepresenting light entertainment, and India over-representing history. The share of local themes was positively related to the country's size of population. In all the countries, questions that awarded higher prizes more frequently required academic knowledge, whereas pocket money questions more often called for popular everyday knowledge. Overall, the global quiz format appears to be sensitive to relatively small cultural differences. The hierarchy of knowledge it features is in line with western norms."

Westerik, Henk et al: The situational and timevarying context of routines in television viewing: an event history amalysis. - S. 155-182

„Building on an action theoretical perspective, it is assumed that most television viewing is a routine response to frequently occurring situations, which together make up everyday life. This interplay between television viewing and everyday life was studied using data from a national survey among Dutch adults ( $\mathrm{n}=$ 825 ) and their families. From this survey, data of 225 couples were analyzed using event history analysis. Results indicate that one cannot see television viewing as merely an alternative for other activities. For instance, participatory activities have two distinct effects: They tend to inhibit television viewing by the actor but stimulate television viewing by the actor's partner. The effect of contacts with other variables appears to be important as well: Being at home, engagement in child care, household work, and eating and drinking often enhances television viewing. But presence of non-family may inhibit television viewing. Education was shown to have a consistently negative impact on television viewing, and there appeared to be some gender specific inducements for termination of television viewing."

Dorer, Johanna: The gendered relationship between journalism and public relations in Austria and Germany: a feminist approach. S. $183-200$

„While journalism traditionally is considered a 'masculine' domain, it is said that public relations are a 'feminine' profession. The legitimation for this gendered coding of two professions are so-called gender different characteristics. The aim of this article is to show how the differentiation of professional roles in journalism and journalism-related fields goes hand in hand with processes of gender differentiating ascriptions on the symbolic and discoursive levels. Addi- tionally, the communication research reproduces these binary codes in context with gender codings. The history of professions shows that gendered practices of ascription are arbitrary and only needed to legitimize gendered positionings in the professional field. A closer look at the gendered positioning of the two professions journalism and public relations shows that both have primary and secondary job markets, horizontal and vertical segmentation, and genderbased differences in pay. The gendered positioning existing on two levels between journalism and public relations as well as within both professions - is not the product of gender different characteristics but of gendered ascriptions of meaning to different job positions. Therefore, it is necessary to analyze both the gendered practices of ascription on the symbolic and the discoursive levels, as well as the gendered positioning in the relationship of journalism and public relations and within both professions. Finally, the consequence for the main/malestream research in the relationship between journalism and public relations could be that the 'feminization' of public relations leads public relations researchers to underestimate the power of public relations."

Peeters, Allerd L.: Bridging or bonding?: Relationships between integration and media use among ethnic minorities in the Netherlands. S. 201-232

„This article will first of all present a brief literature review on media use and identity construction and integration. This overview will be given in light of two phenomena: The concepts of 'social quality' and 'cultural participation' and the role played by the media in this on the one hand, and the multicultural composition of Dutch society on the other. The present contribution looks at the four largest ethnic minority groups in the Netherlands: Turkish, Moroccan, Surinamese and Antillean youngsters (13 to 24-year-olds), and adults ( 25 years of age and above). The key issue is 'integration' with its six dimensions, among which the extent to which ethnic minorities endorse norms and values of Dutch society, and the minorities' motivation with respect to integration. We also focus on related features, such as religious outlook and linguistic fluency. The measure of participation or integration in Dutch society is related to the use of four kinds of media: Radio, television, print media, and the Internet. The present contribution addresses the following question: To what extent are, on the one hand, bonding (i. e., an immigrant's slant towards maintaining contacts with the 'homeland') or, on the other, bridging (i. e., the desire to familiarize oneself with Dutch society and to participate in it socially and culturally) predictors of media use among ethnic minorities? We will address the relative importance of these four media in terms of media time and their twofold 'bridging' and 'bonding' capacities; for each of these media we check the extent to which ethnic minorities favor media from their native country and use applications which focus on their country of origin or their own group."

Minnebo, Jurgen: Psychological distress, perceived social support, and television viewing for reasons of companionship: a test of the compensation hypothesis in a population of crime victims. - S. $233-250$ 
„Becoming a crime victim is often associated with the development of psychological distress symptoms. In turn, these symptoms have been found to be related to a decrease in perceived social support by the victim. From a uses and gratifications point of view, the increase in distress and the decrease in perceived social support could well affect a victim's television use. Furthermore, the compensation hypothesis (Davis and Kraus, 1989) proposes that people with little social contact use mass media to compensate for social isolation. It could therefore be hypothesized that increased use of television for reasons of companionship is related to higher levels of psychological distress and lower levels of perceived social support. The present study used a structural equation model to test this hypothesis. The sample consisted of 212 Flemish victims of crime. The results confirm the proposed relationships and hypotheses. Directions for future research are discussed.

Broos, Agnetha: Marginality in the Information age: is the gender gap really diminishing? -S. 251-260

„Recent research predicts the narrowing of the gender gap concerning new media use. This article presents the results of a quantitative study $(n=1058)$ of the gender gap in Flanders. Significant gender differences were found with men having more access to, and making more use of computers, the Internet and e-mail. In general, females reported more negative attitudes towards new media than men did. Thus, it appears that, despite American research indicating the opposite, in Flanders the gender gap is still very much in evidence. To contextualize the relationship between gender, computer anxiety, and attitudes a multiple regression analysis was carried out on socio-demographic variables and computer-related items. The results showed that, although gender remains a significant factor, it is computer experience which is the strongest predictor of computer anxiety and attitudes."

\section{Computer Law Review International}

\section{Jg 6 (2005) Nr 2}

Joslove, Bradley L.: Dangerous Liaisons: Liability in the European Union for hypertext linking and search engine services. - S. 3339

Beardwood, John P.: Revolution in Canadian Copyright Law?: how the Jurisdiction shifted the Balance between Creator's Rights and User's Rights. - S. 40-47

Feig, Erik M.: Keyword Advertising: why all the Fuss?: an examination of a Lucrative Business Model's Legality. - S. 48-55

\section{Jg 6 (2005) Nr 3}

Pühler, Alexander C.: Will „Grokster“ prevail on the merits of "Sony"?: some thoughts on contributory copyright of infringement and the „Sony Betamax" exemption in consideration of the oral arguments before U.S. Supreme Court on 29 March 2005. - S. 65-70

Blume, Peter: Personal Data Transfer: Possibilities and Limitations. - S. 71-78

\section{Computer und Recht}

\section{Jg 21 (2005) Nr 3}

Lensdorf, Lars: Auslagerungen von IT-Leistungen auf Public Private Partnerships: Privatisierung und Vergaberecht bei der Aufgabenverlagerung auf gemischtwirtschaftliche Unternehmen. - S. 161-168

Wiebe, Andreas: Europäischer Datenbankschutz nach „William Hill“: Kehrtwende zur Informationsfreiheit?. - S. 169-175

Katko, Peter: Voice-over-IP: entscheidender Schritt zur Konvergenz oder nur preiswert Telefonieren. - S. 189-194

Der Beitrag klärt die grundsätzliche Funktionsweise von "Voice-over-IP“ und geht den wirtschaftlichen Chancen sowie den regulatorischen bzw. telekommunikationsrechtlichen Fragen der Internettelefonie nach. Im Rahmen der rechtlichen Erörterungen werden die Nummernzuteilung ( $\$ 66$ TKG), die Verpflichtung zu Notrufmöglichkeiten (\$108 TKG), die Datenschutzregelungen, die technische Umsetzung von Überwachungsmaßnahmen sowie die Entbündelung angesprochen. Kurz wird auf die Konvergenzentwicklung hingewiesen sowie das technische und wirtschaftliche Potential von Voice-over-IP betont.

Heim, Andreas: Zur Markenbenutzung durch Meta-Tags: bisherige Trends und ein neuer differenzierter Ansatz zur Beurteilung markenrechtlicher Zulässigkeit von Meta-Tags. S. $200-205$

Der Beitrag erörtert die markenrechtliche Zulässigkeit von so genannten Meta-Tags. Zunächst wird anhand der Darstellung der unterschiedlichen Auffassungen in die Problematik eingeführt. Im Mittelpunkt stehen sodann eine kritische Besprechung zweier Entscheidungen des OLG Düsseldorf aus den Jahren 2003 und 2004 und die Entwicklung eines neuen, differenzierenden Ansatzes zur Beurteilung der markenrechtlichen Relevanz von Meta-Tags. Der Autor schlägt letztlich eine zweistufige Prüfung zur Ermittlung der Kennzeichenwidrigkeit vor.

\section{Jg 21 (2005) Nr 4}

Sahin, Ali: Einräumung von Nutzungsrechten im gestuften Vertrieb von Standardsoftware: unter welchen Voraussetzungen kann ein Vertragshändler Endkunden Nutzungsrechte einräumen?. - S. 241-246

Gaster, Jens L.: Kartellrecht und geistiges Eigentum: Unüberbrückbare Gegensätze im EG- 
Recht?: Die Auflösung eines klassischen Spannungsverhältnisses im Spiegel der europäischen Rechtsprechung. - S. 247-253

Klees, Andreas: Erneuter Anlauf für mehr Verbraucherschutz bei Mehrwertdiensten: ein Ausblick auf das geplante TKG-Änderungsgesetz. - S. 262-267

Der Beitrag analysiert die bisherigen gesetzgeberischen Maßnahmen sowie die Rechtsprechung der Zivilgerichte $\mathrm{zu}$ Mehrwertdiensten und insbesondere dem Dialer-Problem. Zugleich gibt der Beitrag einen Ausblick auf die geplanten verbraucherschutzrechtlichen Maßnahmen im Rahmen des Entwurfes eines Gesetzes zur Änderung telekommunikationsrechtlicher Vorschriften vom 4.2.2005 (TKG-ÄndG-E). Dazu wird einleitend auch das Spannungsfeld zwischen Innovationsschutz einerseits und Verbraucherschutz andererseits bei Mehrwertdiensten aufgezeigt.

Erdemir, Murad: Jugendschutzprogramme und geschlossene Benutzergruppen: zu den Anforderungen an die Verbreitung entwicklungsbeeinträchtigender und jugendgefährdender Inhalte im Internet. - S. 275-281

Zunächst führt der Autor in die verfassungsrechtlichen Vorgaben und den spezialgesetzlichen Jugendschutz ein. Anschließend wendet sich der Beitrag den Anforderungen des Jugendmedienschutz-Staatsvertrages (JMStV) im Hinblick auf unzulässige und entwicklungsbeeinträchtigende Angebote (\$S 4 und 5 JMStV) zu. Ferner werden die gesetzlichen Anforderungen zu geschlossenen Benutzergruppen $(\mathbb{4} 4 \mathrm{Abs} .2$ S. $2 \mathrm{JMStV})$ und Jugendschutzprogrammen $(\mathbb{S} 5,11$ JMStV) beleuchtet. Letztlich geht der Autor noch auf die Regelungen zum Jugendmedienschutz im Rahmen des Jugendschutzgesetzes (JuSchG), und zwar im Hinblick auf den Internethandel mit Trägermedien und Online-Gewinnspiele, ein.

\section{Jg 21 (2005) Nr 5}

Scheja, Katharina: Vorvertragliche Pflichten bei der privaten Ausschreibung von Outsourcing-Vorhaben: Untersuchung der Anforderungen an die Fairness einer privatrechtlichen Ausschreibung. - S. 321-330

Schütze, Marc: Entgeltkontrolle bei Bereitstellung von TK-Vorleistungen: die Sprengkraft des Übergangs von der Ex-ante- zur Ex-postEntgeltkontrolle. - S. 332-336

Der Autor widmet sich in diesem Beitrag einer Entscheidung des VG Köln aus dem Jahre 2005, das die Entgeltkontrolle bei so genannten Carrier-Festverbindungen (CFV) betraf. Dazu werden die unterschiedlichen Ansätze zur Genehmigungspflicht von CFV-Entgelten beleuchtet und im Hinblick darauf die Entscheidung des VG Köln analysiert. Der Autor weist sodann auf die strategischen Konsequenzen hin und fordert taktische Reaktionen der Bundesnetzagentur (vormals RegTP). Letztlich wird die Frage aufgeworden, ob deren Entscheidungspraxis in den Anwendungsbereich der Amtshaftung fällt.
Rücker, Daniel: Notice and take down-Verfahren für die deutsche Providerhaftung?: zur Begrenzung der Unterlassungshaftung von Online-Diensten durch das „Verbot allgemeiner Überwachungspflichten “. - S. 347-354

Der Beitrag setzt sich kritisch mit einer Entscheidung des BGH aus dem Jahre 2004 (rolex vs. ricardo.de) auseinander. Anhand des europäischen Rechtsrahmens, der sog. E-Commerce-Richtlinie, wird verdeutlicht, dass die Begründung von Unterlassungsansprüchen gegen Provider nicht europarechtskonform ist. Besonderer Aufmerksamkeit widmet der Autor der Begrenzung von Unterlassungsansprüchen über das Verbot allgemeiner Überwachungspflichten. Letztlich wird das sog. „Notice-and-take-down"Verfahren in den Blick genommen und ein Bezug zu den vorangehenden Ausführungen hergestellt.

\section{Jg 21 (2005) Nr 6}

Bechtold, Stefan: Trusted Computing: rechtliche Probleme einer entstehenden Technologie. -S. 393-404

Peter, Stephan: Verfügbarkeitsvereinbarungen beim ASP-Vertrag: Beschreibung der Leistung oder mängelhaftungsbeschränkende Abrede? - S. 404-412

Schmitz, Peter: Inhalte- und Störerverantwortlichkeit bei Telekommunikations- und Telemediendiensten: Anregungen für das geplante neue Telemediengesetz. - S. 420-428

Der Beitrag beleuchtet einige Regelungsmaterien des geplanten Telemediengesetzes: So werden die Haftung für Urheberrechtsverstöße, die Störerhaftung, die Haftung für fremde und eigene Inhalte, die Verantwortlichkeit von Host-Providern durch Kenntnis und die Haftung für Hyperlinks als im Wesentlichen zu berücksichtigende Themenfelder bezeichnet. Die Autoren gehen jeweils von der geltenden Rechtslage und Rechtsprechung aus und zeigen sowohl die Problemkreise, als auch Lösungsalternativen auf. Insgesamt wird die Schaffung von angemessenen Rahmenbedingungen gefordert.

Sobola, Sabine: Haftung von Providern für fremde Inhalte: Haftungsprivilegierung nach $\$ 11$ TDG: Grundsatzanalyse und Tendenzen der Rechtsprechung. - S. 443-450

Die Autoren stellen den Anwendungsbereich des Teledienstegesetzes (TDG) für Plattformbetreiber kurz dar. Anschließend wird erörtert, wie der von der Rechtsprechung normierte Begriff der „zu Eigen gemachten Inhalte“ im neuen TDG gesehen werden könnte. Danach erfolgt eine Auseinandersetzung mit den Voraussetzungen von $\int 11$ TDG (Verantwortlichkeit von Host-Providern), insbesondere des für die Privilegierung entscheidenden Begriffs der „Kenntnis“. In einer vertieften Grundsatz- und Rechtsprechungsanalyse wird die Anwendbarkeit der Haftungsprivilegierung auf Unterlassungsansprüche erörtert und anschließend zu Beweislastfragen Stellung genommen. 


\section{Convergence}

\section{Jg 11 (2005) Nr 1}

Olson, Kathleen K.: Cyberspace as Place and the Limits of Metaphor. - S. 10-18

Lee, Alistair: Disabling Web Designers: issues surrounding disabled people's Use of Web Related Technology. - S. 19-25

Johnson, Phylis: KJLH-FM, South Central's Hub to the World: Black Radio's Emergence on the Internet. - S. 26-47

„KJLH-FM's Peabody Award winning Front Page programme was transformed in 1992 during the Los Angeles civil unrest. Ten years later, the internet provided the impetus for KJLH to present its community issues to a national audience, while continuing its commitment to local listeners. This case study examines how one small community commercial radio station extended its audience through streaming its popular and controversial public affairs programme across the internet. KJLH-FM, with only 5,600 watts of transmitting power, has embraced new technologies in its efforts to extend its audience across the USA and beyond, and to remain competitively and civically engaged in a highly deregulated and consolidated marketplace."

Newman, James: Playing (with) Videogames. S. $48-67$

„This paper examines the practices and activities of videogame fans online. In scrutinising a variety of player-produced texts including walkthroughs, fan art, fiction, 'theorising', Frequently Asked Questions lists (FAQs), and even fan-games, the article seeks not only to highlight the creativity and vibrancy of the participatory culture of videogame fandom but also to examine the ways in which production of such texts and the ensuing discussion and debate are used by players to generate and communicate their identity within the community of 'hardcore' game fans (often known as otaku) and modify the terms of engagement with the game. In this way, the paper interrogates player-produced texts as examples of the involvement and activity of players in the construction of videogames' meaning and as a means of problematising discussions of the pleasures of gameplay."

Kogen, Lauren: The Spanish Film Industry: New Technologies, New Opportunities. S. $68-87$

„Spain, like other countries of Western Europe, has struggled against Hollywood to maintain its national film industry. Most of the films that are produced lose money, and producers are increasingly reliant on state subsidies. This article outlines the potential benefits of digital technologies to Spanish cinema and recommends changes in government film policy to take advantage of these benefits. State subsidies, in Spain as well as elsewhere in Europe, have created a weak national film market. Most Spaniards view national cinema as low-grade, and consistently opt for foreign fare. This article argues that the government should decrease production subsidies in favour of an investment in digital theatre projectors, thus permitting all productions to take advantage of the cost-saving aspects of digital filmmaking. In the course of researching this article, interviews were conducted with among others Bigas Luna (director of Jamon, Jamon, 1992 and La Teta y la Luna, 1994); Tomás Pladevall (President of the Spanish Association of Cinematographers); José-María Aragonés (Artistic Director of Filmtel, the post-production arm of Filmax production company); Ricardo Gil (Marketing and PR Director of UCI / Cinesa theatres in Spain and Portugal); and J. M. Caparrós Lera (Director of the Center for Cinematic Research in Barcelona). All were interviewed about the audience appeal, artistic merit and technical advantages or disadvantages of the digital medium."

Zagal, José Pablo: From Samba Schools to Computer Clubhouses: Cultural Institutions as Learning Environments. - S. 88-105

Punt, Michael: Timeshift: The World in Twenty-Five Years: Ars Electronica 2004 (Linz 2-7 September 2004. - S. 106-113

\section{Communicatio Socialis}

\section{Jg 38 (2005) Nr 2}

Schmolke, Michael: Die kirchliche Publizistik im jüngeren Strukturwandel der Öffentlichkeit. - S. 121-137

Nach dem zweiten Weltkrieg hatte die christliche Presse eine gute Position; auch in den öffentlichrechtlichen Rundfunkanstalten waren die Kirchen gut positioniert. Aber die Strukturen der öffentlichen Kommunikation veränderten sich schneller als die kirchlichen Medien folgen konnten. Nur auf einem Gebiet sind die kirchlichen Medien voll konkurrenzfähig: Während die allgemeinen (säkularen) Medienangebote immer weniger zuverlässig sind, bleibt die Stärke der kirchlichen Medien ihre Glaubwürdigkeit.

Haimerl, Helmut: Zukunftsfähige Bistumszeitungen: konzeptionelle Überlegungen der Medien-Dienstleistungsgesellschaft. - S. 138-153

Mönch-Tegeder, Theo: Umsetzung des Konzepts „Zukunftsfähige Bistumszeitungen“ in der Verlagsgruppe Bistumspresse. - S. 154-157

Hampp, Constanze: Medien im Kloster: eine qualitative Studie zu den Nutzungsmotiven von Ordensleuten. - S. 159-173

„Massenmedien und Religion sind zumindest teilweise funktional äquivalent: Sie können den Alltag strukturieren und das Bedürfnis nach Kontinuität und Vertrautheit befriedigen, sie stellen Verhaltensmodelle und ein Wertesystem bereit. Der Beitrag fragt danach, wie Menschen mit den Angeboten der Massenmedien umgehen, in deren Leben die Ausübung von Religion im Zentrum steht. ... Die Studie zeigt den überragenden Einfluss des Klosteralltags. Den Befragten bleibt wenig Zeit für die Mediennutzung. ... Medieninhalte können die Ordensleute bei der Erfüllung ihrer Pflichten unterstützen, sie dienen der Rekreation und 
bieten einen Ausgleich zum verzichtreichen Alltag, sie schaffen gemeinsame Bezugspunkte und helfen manchmal sogar bei der Identitätsbildung."

Theobald, Adolf: Heiko Klinge: höher, schneller und so weiter: zur Verabschiedung des Geschäftsführers der MDG. - S. 174-179

Kopp, Matthias: Für eine gelungene Kommunikation: Kommunikationskonzept des XX. Weltjugendtages 2005 in Köln. - S. 180-197

Nicolini, Markus: Nadelöhr journalistischer Berufseinstieg: gute Chancen für Stipendiaten des Instituts zur Förderung publizistischen Nachwuchses. - S. 198-212

Rude, Bernhard: Fit werden für den Redaktionsalltag: 15 Jahre Ausbildungs-Tarifvertrag für Tageszeitungsvolontäre. - S. 213-222

Streier, Eva-Maria: Kirchengeschichte spannend verkauft: der Communicator-Preis für Hubert Wolf. - S. 223-228

\section{European Journal of Communication}

\section{Jg 20 (2005) Nr 2}

Erjavec, Karmen: Hybrid Public Relations News Discourse. - S. 155-180

In Slowenien lässt sich wie in anderen mittel- und osteuropäischen Ländern ein Bedeutungszuwachs von Public Relations erkennen. Der Beitrag stellt eine Studie vor, die das Vordringen von PR in die Nachrichtenproduktion Sloweniens mittels Diskursanalyse sowie einer so genannten ,interpractice analysis“ untersuchte. Methodisch wurde eine Textanalyse mit einer Analyse des Produktionsprozesses (durch teilnehmende Beobachtung und Tiefeninterviews) kombiniert. Als wesentliche Faktoren für die große Bedeutung, die PR-Informationen für die Nachrichtenproduktion haben, fand Erjavec fünf Merkmale: Die einfache Zugänglichkeit von Informationen über PRAbteilungen, Presse-Konferenzen etc.; journalistische Routinen; ökonomische, politische, kulturelle und ideologische Ausübung von Druck; Mangel an $\mathrm{Hu}-$ manressourcen und Zeitdruck in den Redaktionen; persönliche Beziehungen von Journalisten und Redakteuren.

Kuhn, Raymond: „Be very afraid“: Television and „l'Insécurité “ in the 2002 French Presidential election. - S. 181-198

Raeymaeckers, Karin: Letters to the editor: a feedback opportunity turned into a marketing tool: an account of selection and editing practice in the Flemish Daily Press. - S. 199-222

Boyle, Raymond: „A small country with a big ambition“: Representations of Portugal and England in Euro 2004 British and Portuguese Newspaper Coverage. - S. 223-244

Der Artikel untersucht die Art der Darstellung Portu- gals bzw. der portugiesischen Kultur und Gesellschaft in der britischen und portugiesischen Zeitungsberichterstattung über die Fußball-Europameisterschaft 2004 (EM). Eines der Ergebnisse ist die Beobachtung, dass nationale Medien internationale Medienereignisse durch nationale Frames reflektieren: Während in Portugal die EM als Möglichkeit der RePositionierung des Landes in der europäischen Staatenwelt gesehen wurde, erschien Portugal in der englischen Berichterstattung ausschließlich als eher rückständiges Urlaubsziel, demgegenüber England als modernes, fortschrittliches Land erschien.

Schönbach, Klaus: Online and Print newspapers: their impact on the perceived public agenda. - S. 245-258

Der Beitrag stellt die Ergebnisse einer repräsentativen Befragung $(n=1000)$ in den Niederlanden vor, die der Frage nachging, inwiefern die Themenagenda von Online-Zeitungslesern sich von der der Leser gedruckter Zeitungen unterscheidet. Ausgangspunkt war die Feststellung, dass „klassische“ Zeitungsleser ein größeres Spektrum an Themen der Öffentlichkeit wahrnehmen als Nutzer anderer Medien. Für die Nutzung von Online-Zeitungen wird demgegenüber angenommen, dass durch die aktivere Rezeption, die enger an den eigenen Interessen orientiert ist, das Spektrum wahrgenommener Themen geringer ist als das der Leser der Printausgaben. Die Ergebnisse sind etwas differenzierter: Bei beiden Gruppen von Zeitungslesern ließ sich eine Erweiterung der Agenda feststellen, wobei dieser Effekt bei den OnlineZeitungslesern nur in Gruppen höherer Bildung erkennbar war, während bei Lesern gedruckter Zeitungen die "Horizonterweiterung“ am größten war bei Personen mit durchschnittlichem Themeninteressen.

\section{Federal Communications Law Journal}

\section{Jg 57 (2004) Nr 2}

Communications Policy and the Second Bush Administration. - S. 167-242

Reiter, Harvey: The Contrasting Policies of the FCC and FERC Regarding the Importance of Open Transmission Networks in Downstream Competitive Markets. - S. 243-324

Kaplan, Debra: Broadcast Flags and the War Against Digital Television Piracy: a Solution or Dilema for the Digital Era?. - S. 325-344

\section{Javnost}

\section{Jg 12 (2005) Nr 2}

Kömecoglu, Ugur: The publicness and sociabilities of the Ottoman Coffeehouse. - S. 5-22

Gunaratne, Shelton A.: Asian philosophies and authoritarian press practice: a remarkable contradiction. - S. 23-38 
Mosco, Vincent: Here Today, Outsourced Tomorrow: Knowledge Workers in the Global Economy. - S. 39-56

Brüggemann, Michael: How the EU constructs the European Public Sphere: Seven strategies of Information Policy. - S. 57-74

"If there is no such thing as a European Public Sphere (EPS), why don't we construct one? The answer seems to be obvious: there is no way one could construct a public sphere top-down since it depends on the active participation of speakers, the media and audience. In a democratic society they are free to deliberate with whom and about what they want. This article does not challenge the Habermasian notion of a public sphere evolving from the free discourse of the citizens. Nevertheless, the evolution of a public sphere is also structured by incentives and constraints imposed from above. The European Union structures the EPS - as a polity as well as through its policies and politics. While it is true that different policies such as media policy and all cultural policies matter for the public sphere, this paper concentrates on the Commission's information policy as it constitutes the most direct link between the institution and the EPS. Seven different strategies of information policy will be presented which vary in their potential of creating or suppressing the evolution of a democratic public sphere. The extremes are marked by propaganda and arcane policy on the one hand and dialogue and transparency on the other hand. While the Commission pursued arcane policies for a long time, its approach to information has changed during the last decade. A change of paradigm might be under way but the legacy of European policy without 'Öffentlichkeit' constraints all attempts at pursuing more democratic information policies aimed at strengthening the public sphere."

Erbe, Jessica: „What do the papers say?": how press reviews link national media arenas in Europe. - S. 75-92

Wimmer, Jeffrey: Counter-Public Spheres and the Revival of the European Public Sphere. S. 93-110

\section{Journal of Communication}

\section{Jg 55 (2005) Nr 1}

Wood Aleman, Melissa: Embracing and Resisting Romantic Fantasies as the Rhetorical Vision on a SeniorNet Discussion Board. S. 5-21

Anker, Elisabeth: Villains, Victims and Heroes: Melodrama, Media, and September 11. S. 22-37

Bloch, Linda-Renée: „I know I'm a Freierit, but ...": how a Key Cultural Frame (en)Genders a discourse of Inequality. - S. 38-55

Frith, Katherine: The Construction of beauty: a cross-cultural analysis of women's magazine advertising. - S. 56-70

Hardy, Bruce W.: Examining Differential Gains from Internet Use: Comparing the Moderating Role of Talk and Online Interactions. S. 71-84

Holbert, Lance: Debate Viewing as Mediator and Partisan Reinforcement in the Relationship Between News Use and Vote Choice. -S. 85-102 Kim, Sei-Hill: Who Cares About the Issues?: Issue Voting and the Role of News Media During the 2000 U.S. Presidential Election. S. $103-121$

Knobloch, Silvia et al: Children's Sex-Stereotyped Self-Socialization Through Selective Exposure to Entertainment: Cross-Cultural Experiments in Germany, China, and the United States. - S. 122-138

Lauf, Edmund: National Diversity of Major International Journals in the Field of Communication. - S. 139-151

The journal ranking of the Institute for Scientific Information (ISI) is increasingly turning into an international currency for the quality of research output. More than 40 communication journals are ISI-ranked and thus labelled "major international“ journals. This analysis of ISI data reveals that the attribute „international" is not always appropriate. National diversity of communication journals is very low due to a dominance of authors from English-speaking countries and U.S. authors in particular. Younger journals and journals with an explicitly stated international mission tend to be international, whereas the internationality of the affiliated organization or impact of a journal had no influence on national diversity. The results suggest that it may be desirable to clearly distinguish between national and international communication journals, to increase the number of international communication journals, and to support authors whose mother tongue is not English.

Len-Rios, Maria E. et al: Representation of Women in News and Photos: Comparing Content to Perceptions. - S. 152-168

Xenos, Michael A.: Politics as Usual, or Politics Unusual?: Position Taking and Dialogue on Campaign Websites in the 2002 U.S. Elections. -S. 169-185

\section{Journal of Health Communication}

\section{Jg 10 (2005) Nr 2}

Wanzer, Melissa: „If we didn't use Humor we'd cry “: humorous coping communication in Health Care Settings. - S. 105-126

Terry-McElrath, Yvonne et al: The effect of 
Anti-Smoking Advertisement Executional Characteristics on Youth Comprehension, Appraisal, Recall, and Engagement. - S. 127-144

Hoffmann-Goetz, Laurie: HIV/AIDS Risk Factors as Portrayed in Mass Media Targeting First nations, Métis, and Inuit Peoples of Canada. - S. $145-162$

Hennink, Monique: Using Research to Inform Health Policy: Barriers and Strategies in Developing Countries. - S. 163-180

Evans, W. Douglas: Evaluating the truth brand. -S. 181-192

\section{Jg 10 (2005) Nr 3}

Engelman, Kimberly K. et al: Cancer Information Needs in Rural Areas. - S. 199-208

Macias, Wendy: Health-Related Message Boards / Chat Rooms on the Web: Discussion Content and Implications for Pharmaceutical Sponsorships. - S. 209-224

Anfang 2000 erteilte das, Health and Human Services' einem Pharmaunternehmen die Erlaubnis, einen gesundheitsbezogenen Chatroom zu sponsern. Die Autorinnen sahen u. a. hierin einen Anlass, sich eingehender mit der Relevanz des Internet für die Gesundheitskommunikation zu befassen. Im Mittelpunkt steht eine explorative Inhaltsanalyse von Mitteilungsbrettern auf verschiedenen Internetseiten zu unterschiedlichen gesundheitsbezogenen Themen.

Farr, A. Celeste et al: The Effectiveness of Media Use in Health Education: Evaluation of an HIV/AIDS Television Campaigns in Ethiopia. -S. 225-236

Der Erfolg gesundheitsfördernder Angebote hängt zuvorderst davon ab, dass sie von der Zielgruppe auch genutzt werden. Die Autorinnen stellen ein Konzept vor, mittels dessen die Nutzung einer in Äthiopien ausgestrahlten Entertainment-Education Radio Soap "The Journey of Life" untersucht bzw. evaluiert wurde. Die Ergebnisse der Studie bescheinigen dem Radioangebot eine hohe Attraktivität und Nutzung. Zudem gab das Gros der Befragten an, dass ihr Gesundheitsverhalten durch die Sendung beeinflusst wurde.

Feeley, Thomas Hugh: Examining College Students' Intensions to Become Organ Donors. S. $237-250$

Knapp Whittier, David et al: Embedding Health Messages into Entertainment Television: Effect on Gay Men's Response to a Syphilis Outbreak. - S. 251-260

Ausgangspunkt der vorgestellten Studie stellte eine hohe Verbreitung von Syphilis unter homosexuellen Männern dar. Im Rahmen einer Gesundheitskampagne wurden in 2003 zwei Episoden in die Krankenhausserie Emergency Room (ER) integriert, die sich speziell diesem Thema widmeten und auf die Bedeu- tung frühzeitiger Behandlung hinwiesen. Die Autoren und Autorinnen untersuchten im Rahmen einer Befragung, inwieweit sich die Rezeption von ER auf die Bereitschaft auswirkte, sich testen zu lassen oder andere $\mathrm{zu}$ einem solchen Test zu motivieren. Ingesamt zeigte sich, dass die Platzierung des Themas in der populären TV-Serie zu einer deutlichen Sensibilisierung der Zielgruppe für dieses Gesundheitsthema führte und die Bereitschaft erhöhte, an frühzeitigen Testverfahren teilzunehmen.

Krugman, Dean M. et al: Understanding the Role of Cigarette Promotion and Youth Smoking in a Changing Marketing Environment. S. $261-278$

\section{Jg 10 (2005) Nr 4}

Forum ; The Life and Work of Everett Rogers: some personal reflections. - S. 285-308

Rose, Abigail et al: The Association Between Knowledge and Attitudes About Genetic Testing for Cancer Risk in the United States. S. 309-322

Johnson, J. David et al: Genomics: the Perfect Information-Seeking Research Problem. S. $323-330$

Stern, Susannah R.: Messages from Teens on the Big Screen: Smoking, Drinking, and Drug Use in Teen-Centered Films. - S. 331-346

Der Beitrag präsentiert Ergebnisse einer Studie, die den Suchtmittelkonsum (Tabak, Alkohol, illegale Drogen) von Jugendlichen in erfolgreichen Jugendfilmen aus den Jahren 1999 bis 2001 untersuchte. Vor dem Hintergrund der Theorie des sozialen Lernens richtete sich der Fokus insbesondere auf das Konsumverhalten der jugendlichen Hauptfiguren $(n=146)$ als potenzielle Identifikationsfiguren und handlungsleitende Modelle. Die Ergebnisse zeigen: Zwei Fünftel der jugendlichen Charaktere konsumierten in den Filmen Alkohol, ein Sechstel Zigaretten, und ein Siebtel Drogen. Die Ergebnisse zeigten jedoch keine signifikanten Unterschiede zwischen den konsumierenden und nicht-konsumierenden Charakteren. Eine besondere Modellwirkung ist insofern von den konsumierenden Charakteren nicht zu erwarten. Stern vermutet jedoch, dass aus kultivierungstheoretischer Perspektive bei jugendlichen Rezipienten der Eindruck entstehen könnte, dass Suchtmittelkonsum im Jugendalter selbstverständlich und weitestgehend problemlos sei.

Mohammed, Shaheed N.: HIV/AIDS Stories on the World Wide Web and Transformation Perspective. - S. 347-360

Pillsbury, Barbara: Women Connect!: Strengthening Communications to Meet Sexual and Reproductive Health Challenges. S. $361-372$ 


\section{Journal of Media Economics}

\section{Jg 18 (2005) Nr 2}

Stephan, Michael: Diversification strategy of global media conglomerates: a comment. S. $85-104$

„Chan-Olmsted and Chang's (2003) framework for the analysis of product-diversification strategies of media conglomerates is critiqued from a dynamic resource-based perspective. In the spirit of spurring intellectual debate, this article proposes a revised framework that incorporates newer findings about the benefits of related product-diversification strategies from the strategic management literature. A differentiated view of the 'media content' notion is presented that distinguishes between assets, skills, and capabilities and competencies. From this differentiated perspective, I argue that there are 2 types of scope effects: traditional economies of scope that grant the media firm a short-term competitive advantage over rivals and dynamic scope effects that contribute to a sustainable competitive advantage." Dem Artikel schließt sich auf den Seiten 105-107 eine Antwort von Sylvia M. ChanOlmstedt an.

Kranenburg, Hans von; Hogenbirk, Annelies: Multimedia, Entertainment, and business software copyright piracy: a cross-national study.S. $109-130$

"This article examines cross-national variations in piracy of U.S. copyright-related products in the multimedia, entertainment, and software industry. To determine which economic, legal, and social factors cause the considerable differences in piracy of U.S. copyright industries' products in individual countries, we tested 4 industry models. We found that for most industries piracy can be explained by the risk profile of the country involved (signaling economic and political stability and growth potential) and the existence of a strong intellectual property rights system. Furthermore, for the 4 analyzed copyright-based industries, considerable variation in piracy exists between geographic regions. This study shows that disaggregation of the copyright piracy data by industry is helpful in analyzing and understanding piracy.”

Chyi, Hsiang Iris: Willingness to pay for online news: an empirical study on the viability of the subscription model. - S. 131-142

„As no business models seem to generate reliable revenue streams for online news services, many publishers see the subscription model as a last resort for survival although little evidence suggests users are ready to pay for online news at this moment. A randomsample telephone survey of 853 Hong Kong residents found very few users actually responded to paid content and most had no intent to pay in the future. Hierarchical regression analysis showed that age and newspaper use are related to paying intent, whereas income is not. Online publishers may consider the economic implications before adopting the subscription model.“

Wurff, Richard van der: Business magazine market performance: magazines for the agriculture, business services, and transportation sectors in the Netherlands. - S. 143-160
„Business magazines provide need-to-know information to decision makers and professionals. Prices, product variety, and diversity of those magazines follow from publishers' differentiation strategies, which in turn depend on market characteristics. Competition and concentration stimulate differentiation strategies, whereas they have opposite effects on prices. However, cost structures and demand conditions make differentiation strategies dependent on the willingness of audiences to pay high subscription prices. Also, noncommercial publishers adopt strategies other than commercial ones. In combination, these factors determine prices, product variety, and magazine diversity in markets for agricultural, business services, and transportation magazines in the Netherlands in the 1990s."

\section{Journalism \& Mass Communication Quarterly}

\section{Jg 81 (2004) Nr 4}

Yang, Hyeseung: Exploring the effects of online advertising on Readers' Perceptions of online news. - S. 733-749

Eine experimentelle Untersuchung zeigt, dass bei den Nutzern mit unterdurchschnittlicher Internet-Erfahrung die Beurteilung des Nachrichtenwertes von Online-Nachrichten durch die Präsenz von Online-Werbung beeinflusst wird.

Park, Sung-Yeon: The effects of brand familiarity in alignment advertising. - S. 750-765

Tsao, James C.: Readership for Free Community papers as a Source of Advertising Information: a Uses-and Gratifications Perspective. S. 766-787

Huh, Jisu: The Information Utility of DTC Prescription Drug Advertising. - S. 788-806

Wood, Michelle L. M. et al: Tonight's Top Story: Commercial Content in Television News. S. $807-822$

Eine explorative Untersuchung nationaler und lokaler Nachrichten in den Kanälen von fünf US-FernsehNetworks und von CNN ergibt, dass ein erheblicher Anteil werbender Inhalte (unbezahlte Marken- oder Firmeninformation oder Verweisungen auf andere Sendungen und Dienste der Rundfunkstation) festzustellen ist, insbesondere in den lokalen Nachrichten.

Reichert, Tom: An Update on Sex in Magazine Advertising: 1983 to 2003. - S. 823-837

Singer, Jane B.: More than Ink-Stained Wretches: the Resocialization of Print Journalists in Converged Newsrooms. - S. 838-856

Reinemann, Carsten: Routine Reliance Revisited: Exploring Media Importance for German Political Journalists. - S. 857-876

Nisbet, Matthew C.: Political Talk as a Catalyst for Online Citizenship. - S. 877-896 
Fico, Frederick: Partisan and Structural Balance of Local Television Election Coverage of Incumbent and Open Gubernatorial Elections. - S. 897-910

Kaye, Barbara K.: Talking a „Blue“ Streak: Context and Offensive Language in Prime Time Network Television Programs. S. 911-928

\section{Jg 82 (2005) Nr 1}

Christen, Cindy T.: The Utility of Coorientational Variables as Predictors of Willingness to Negotiate. - S. 7-24

Park, Hyun Soon: A Test of the Third-PersonEffect in Public Relations: Application of Social Comparison Theory. - S. 25-43

Spaulding, Stacy: Did Women Listen to News?: A Critical Examination of Landmark Radio Audience Research (1935-1948). S. $44-61$

Der Beitrag zeigt, dass die Betonung ganz unterschiedlicher Programmvorlieben von Frauen und Männern in den frühen Studien zur Hörfunknutzung nicht aus den publizierten Daten dieser Untersuchungen abzuleiten ist.

Hardin, Marie: Stopped at the Gate: Women's Sports, „Reader Interest“, and Decision Making by Editors. - S. 62-77

Luther, Catherine A.: Framing of the 2003 U.S.-Iraq War Demonstrations: an Analysis of News and Partisan Texts. - S. 78-96

Fox, Julia R.: Hype versus Substance in Network Television Coverage of Presidential Election Campaigns. - S. 97-109

Mastro, Dana E.: Latino Representation on Primetime Television. - S. 110-130

Uhm, Kiyul: The Cold War Communication Crisis: the Right to Know Movement. S. 131-147

Mizuno, Takeya: Federal Government Uses of the Japanese-language Press from Pearl Harbour to Mass Incarceration. - S. 148-166

Rowley, Karen M.: There's a New Gatekeeper in Town: How Statewide Public Affairs Television Creates the Potential for an Altered Media Model. - S. 167-180

Knobloch-Westerwick, Silvia et al: Selective exposure effects for Positive and Negative News: Testing the Robustness of the Informational Utility Model. - S. 181-196

\section{Kommunikation \& Recht}

\section{Jg 8 (2005) Nr 4}

Ladeur, Karl-Heinz: Rechtsprobleme der Werbung im lokalen Fernsehen. - S. 145-153

Der Beitrag greift die wirtschaftlich schwierige Lage der lokalen Fernsehveranstalter in Deutschland auf und stellt die Rechtslage für die wichtigste Erlösquelle der privaten Veranstalter, der Werbung, dar. Es wird an $\$ 46 \mathrm{a} S \mathrm{RtV}$ angeknüpft, der eine partielle Liberalisierung für lokale Veranstalter schafft, indem im Vergleich zu bundesweiten Programmen eine deutlich flexiblere Berechnung der begrenzten Werbezeiten ermöglicht und u. a. etwa die Einzelspotwerbung erlaubt wird. Der Verfasser kommt zum Ergebnis, dass die Lockerung einiger Werbevorschriften allein nicht ausreichend sei. Er untersucht sodann, ob den Rundfunkgesetzgeber eine verfassungsrechtliche Pflicht zur "Nachbesserung“ der finanziellen Rahmenbedingungen treffen könnte. Vor dem Hintergrund der schleichenden Durchbrechung des Trennungsgebots von Werbung und redaktionellen Inhalten, etwa durch die neue Programmform der „Infomercials“ und der zu erwartenden Konvergenz von TV und Internet zu einem Empfangsgerät, sei der Medienaufsicht ein neuer Konkretisierungsspielraum durch die Ermächtigung zum Richtlinienerlass einzuräumen. Dann erschiene es etwa nach Ansicht des Autors möglich, auf lokaler Ebene „Infomercials" zur Finanzierung von Fernsehprogrammen zuzulassen.

Gosse, Gesa Marisa: Mutmaßung statt Gewissheit: die Zunahme gesetzlicher Vermutungen im Wirtschaftsrecht am Beispiel der $\$ \mathbb{2 2 8 , 4 2}$ TKG. - S. 154-160

Khorrami, Esfandiar: Polit-Spam?: zur rechtlichen Zulässigkeit politischer E-Mail/E-CardWerbung. - S. 161-166

„Politische Werbung erfolgt zunehmend auch unter Einsatz moderner elektronischer Kommunikationsformen. Insbesondere E-Mails und elektronische Grußkarten bieten den Parteien eine einfache und günstige Möglichkeit potenzielle Wähler zu umwerben. Für den Bürger als Adressaten solcher Werbesendungen ist von Interesse, ob gegen die Zusendung ein zivilrechtlicher Unterlassungsanspruch besteht. Rechtliche Aspekte eines solchen Anspruchs sind die Fragen, ob die jeweilige Partei als Störer handelt, inwieweit die politische Werbung im konkreten Fall einen widerrechtlichen Eingriff in das allgemeine Persönlichkeitsrecht des Adressaten darstellt und ob ihm die Zusendung politischer Werbung nicht jedenfalls zumutbar ist." Der Autor kommt zum Ergebnis, dass die Zusendung grundsätzlich zulässig sei. Es bestehe kein Unterlassungsanspruch, da die Parteien zwar als Störer handelten und in das Allgemeine Persönlichkeitsrecht des Empfängers eingriffen, die Handlungen aber nicht widerrechtlich seien. Die gebotene Abwägungsentscheidung zwischen den betroffenen Verfassungsgütern des Empfängers (Allgemeinen Persönlichkeitsrecht, Art. 2 I i. V. m. 1 I GG) und der Parteien (Vereinigungsfreiheit (Art. 9 GG) und Verankerung der Parteien im GG (Art. 21 GG)) sei aufgrund des verhältnismäßig geringen Eingriffs in das Persön- 
lichkeitsrecht des Empfängers zugunsten der Parteienwerbung zu treffen.

Keller, Moritz: Versandhandelskauf und Preisirrtum im Internet. - S. 167-173

\section{Jg 8 (2005) Nr 5}

Körber, Torsten: Meilenstein oder Pyrrhussieg?: zur Entscheidung Microsoft/Kommission des EuG. - S. 193-197

„Das wettbewerbliche Verhalten der Microsoft Corporation beschäftigt seit geraumer Zeit Kartellbehörden und Gerichte diesseits wie jenseits des Atlantiks. Am 24.3.2004 hat die Europäische Kommission eine spektakuläre, kartellrechtliche Missbrauchsverfügung gegen Microsoft erlassen. Microsoft hat diese Verfügung vor dem Europäischen Gerichtshof angegriffen. $\mathrm{Da}$ sich das Hauptsacheverfahren noch über Jahre hinziehen dürfte, hat es zusätzlich einstweiligen Rechtsschutz beantragt. Damit ist es am 22.12.2004 vor dem Europäischen Gericht erster Instanz (EuG) gescheitert. Der Beitrag beschreibt und bewertet Hintergründe, Verfahren und wesentliche Inhalte des umfangreichen und bisher nur in englischer und französischer Sprache im Internet verfügbaren Beschlusses des Präsidenten des EuG.“"

Ladeur, Karl-Heinz: Das europäische Telekommunikationsrecht im Jahre 2004. - S. 198207

Der Beitrag gibt einen Überblick über wichtige Entscheidungen und Mitteilungen der Kommission und die Rechtsprechung europäischer Gerichte, die für das Telekommunikationsrecht von Bedeutung sind. Dabei bildet ein Schwerpunkt die Umsetzung des Richtlinienpakets, insbesondere das Art.-7-Verfahren, in die Praxis der Mitgliedstaaten und der EG. Zudem werden Mitteilungen und Stellungnahmen der Kommission u. a. zur Frequenzpolitik und den elektronischen Märkten sowie Wettbewerbsentscheidungen zusammengefasst.

Bosch, Tobias: Alter Wein in neuen Schläuchen?: dürfen Zusammenschaltungsentgelte nicht marktmächtiger Unternehmen unter den neuen TKG reguliert werden?. - S. 208-217

„Bereits unter dem bisherigen TKG (,TKG-1996") war eines der zentralen und ungelösten Probleme die Entgeltregulierung nicht marktmächtiger Unternehmen. Diese seit In-Kraft-Treten des TKG-1996 umstrittene Frage wurde in jüngerer Zeit durch die Entscheidungen der Regulierungsbehörde für Telekommunikation und Post (,RegTP') zu nichtreziproken Entgelten sowie die sich daran anschließenden $\mathrm{Ge}-$ richtsentscheidungen erstmals auch in praktischer Sicht bedeutsam, ohne dass sie allerdings einer abschließenden Lösung zugeführt wurde. Diese Frage stellt sich unter dem am 26.6.2004 in Kraft getretenen neuen TKG selbstverständlich weiterhin. Dabei ist zu erwarten, dass dieses Problem - wie die Anzahl und Vielfalt der derzeit bereits unter neuem Recht dazu anhängigen Verfahren zeigt - in Zukunft noch einen größeren Raum einnehmen wird, da das TKG in $\$ \mathbb{S} 18,25$ TKG dezidiert eine Regulierung nicht marktmächtiger Unternehmen, die den Zugang zu Endnutzern kontrollieren, vorsieht. Dafür spricht auch, dass sich der regulatorische Fokus in Zukunft aufgrund der fortschreitenden Liberalisierung mehr auf bilaterale Zusammenschaltungsverhältnisse nicht marktmächtiger Betreiber richten wird."

Baumann, Rico: Entbündelter Zugang zu Netzelementen: Entwicklungen in der USA. S. $218-221$

„Dieser Beitrag gibt einen Überblick über die jüngsten Entwicklungen in den USA betreffend die Regulierung des entbündelten Zugangs zu Netzwerkelementen. Für Europa ist die Entwicklung in der USA interessant, weil daraus Rückschlüsse auf mögliche zukünftige Regulierungsentscheidungen von Gemeinschaftsorganen oder nationalen Regulierungsbehörden gezogen werden können. Gemeinsamer Hintergrund sind die in den USA und in Europa gleichermaßen umstrittenen Fragen: Welche Netzwerkelemente müssen entbündelt angeboten werden und zu welchem Preis ?"

Baumann, Rico: Zum Auskunftsanspruch nach \101a UrhG. - S. 222-224

\section{Jg 8 (2005) Nr 6}

Krüger, Thomas: Nebenentgelte in Mobilfunkverträgen: Überprüfbarkeit und Rechtmäßigkeit. - S. 241-251

Mayer, Christoph: Bekämpfung von Spam mit den Mitteln des Telekommunikationsrechts durch die RegTP. - S. 251-258

"Jahrelang lag das Vorgehen gegen unverlangt zugesandte Werbung in der Hand der Betroffenen und von Verbänden. Die allseits bekannten damit verbundenen Probleme, wie z. B. die Identifizierung des Versenders, Fragen der Klagebefugnis, Verantwortlicher befindet sich im Ausland, trugen nicht unbedingt zu einer effektiven Spam-Bekämpfung bei. Auch war aufgrund der naturgemäß undurchführbaren Koordinierung der mit der Spam-Bekämpfung Befassten kein systematisches Vorgehen möglich. Im August 2003 trat das Gesetz zur Bekämpfung des Missbrauchs von 0190er-/0900er-Mehrwertdiensterufnummern mit verschiedenen Vorgaben zu 0190er-/0900er-Mehrwertdiensterufnummern in Kraft. Eine ausdrückliche Anti-Spam-Vorschrift fehlte jedoch. Daran änderte auch die tiefgreifende TKG-Novelle im Juni 2004 nichts. Gleichwohl gibt die Ermächtigungsgrundlage des \$67 TKG der RegTP die Möglichkeit, gegen unverlangt zugesandte Werbung vorzugehen. Voraussetzung ist jedoch, dass der Spam im Zusammenhang mit einer Rufnummer erfolgt (Rufnummernspam). Somit gibt es in Deutschland erstmals die Möglichkeit für eine staatliche Stelle systematisch und flächendeckend die Spam-Flut einzudämmen."

Ditscheid, Alexander: Das Verhältnis von \305a Nr 2 lit. b BGB zu den Informationspflichten im Fernabsatz- und E-CommerceRecht. - S. 258-263

Zscherpe, Kerstin A.: Datenschutz im Internet: Grundsätze und Gestaltungsmöglichkeiten für Datenschutzerklärungen. - S. 264-269 
Cornelius, Kai: Strafrechtliche Grenzen der zentralen E-Mail-Filterung und -Blockade. S. $269-271$

\section{Jg 8 (2005) Nr 7}

Klett, Alexander R.: Die Entwicklung des Urheberrechts im Jahr 2004. - S. 289-294

„[...] Dieser Beitrag beleuchtet die Entwicklung des Urheberrechts im Jahr 2004, in welchem erstmals seit 1992 keinerlei Änderung des Urheberrechtsgesetzes erfolgt ist. Die nächste Urheberrechtsnovelle (der so genannte „zweite Korb“) wird jedoch bereits heftig diskutiert und hat die deutsche Urheberrechtspraxis im vergangenen Jahr nicht wenig beschäftigt. Auch die höchst- und obergerichtliche Rechtsprechung zum Urheberrecht im letzten Jahr hat erneut einen erheblichen Umfang."

Degenhart, Christoph: Öffentlich-rechtlicher Rundfunkauftrag und Rundfunkgebühr nach dem siebten und achten Rundfunkänderungsstaatsvertrag. - S. 295-301

„Mit dem achten Rundfunkänderungsstaatsvertrag bleiben die Landtage erstmals hinter dem Gebührenvorschlag der KEF zurück. Deshalb, wie auch im Hinblick auf sein Zustandekommen, sieht sich der Staatsvertrag verfassungsrechtlichen Angriffen ausgesetzt, die jedoch nach Ansicht des Verfassers unbegründet sind. $\mathrm{Zu}$ begrüßen seien Ansätze zu einer staatsvertraglichen Konkretisierung des öffentlich-rechtlichen Rundfunkauftrags, zumal die Forderung des Gemeinschaftsrechts nach Klarheit, Transparenz und Kontrolle im Raum steht."

Szczesny, Michael: Zur Unternehmereigenschaft und ihren zivilrechtlichen Folgen im Rahmen von Internetauktionen. - S. 302-307

Bach, Florian: Zahlungen über Mobilfunknetze (M-Commerce). - S. 308-312

Paschke, Mirko: Rufnummernnutzung für Voice-over-Internet-Protocol. - S. 313-317

„Im Jahr 2004 wurde seitens der RegTP eine Reihe von Ânderungen am deutschen Rufnummernplan eingeleitet, um diesen im Sinne technologieneutraler Regulierung an die Anforderungen der zunehmenden Verwendung IP-basierter Netzstrukturen für Sprachtelefoniedienste anzupassen. Der Beitrag, der auf einem vom Autor am 1.3.2005 anlässlich des 4. ENUMTages der DENIC eG gehaltenen Vortrag basiert, gibt zunächst einen Überblick über die Ausgangslage und erläutert dann die im Laufe des Jahres 2004 eingeleiteten Maßnahmen der RegTP. Ausgehend vom status quo zeigt der Artikel schließlich die noch ausstehenden Entscheidungen auf."

\section{Mass Communication \& Society}

\section{Jg 8 (2005) Nr 2}

Pardun, Carol J. et al: Linking exposure to outcomes: early adolescents' consumption of sexual content in six media. - S. 75-92
Rojas, Hernando et al: Media dialogue: perceiving and adressing community problems. S. $93-110$

In einem quasi-experimentellen Design wurden Fernsehzuschauer für unterschiedliche Diskussionsrunden über den Dokumentarfilm "Two Tows of Jasper" rekrutiert, in dem es um die Auseinandersetzungen in Jasper, Texas, nach einem rassistisch motivierten Mord geht. Es zeigt sich, dass die Bereitschaft, dieses Thema zu diskutieren und sich politisch zu beteiligen, mit der Mediennutzung zusammenhängt, besonders aber mit der bereits erfolgten Teilnahme an einer heterogen zusammengesetzten Diskussion über diese Dokumentation.

Moy, Patricia: Communication and citizenship: mapping the political effects of Infotainment. - S. 111-132

Kim, Sei-Hill: Media use and participatory democracy in South Korea. - S. 133-154

Billings, Andrew C.: Diverging discourses: gender differences in televised golf announcing. -S. 155-172

\section{Media Asia}

\section{Jg 31 (2004) Nr 3}

Adhikarya, Ronny: A personal tribute to Everett Rogers. - S. 123-127

Navarro, Rex L.: Reaching the Unreached. S. $128-132$

Hwa, Ang Peng: A model of Internet Rule Development: a case study of liability for ThirdParty Content. - S. 133-140

\section{Media Culture \& Society}

\section{Jg 27 (2005) Nr 2}

Curtin, Michael: Murdoch's dilemma, or "What's the price of TV in China?". S. 155-176

Leyshon, Andrew et al: On the reproduction of the musical economy after the Internet. S. $177-210$

Alhassan, Amin: Market valorization in broadcasting policy in Ghana: abandoning the quest for media democratization. - S. 211-228

Henning, Victor; Alpar, Andre: Public aid mechanism in feature film production: the EU MEDIA Plus Progamme. - S. 229-250

2002 wurde das Förderprogramm der EU für die europäische Filmindustrie - MEDIA - mit einer dritten Ausschreibung verlängert. Trotz 12-jähriger Laufzeit konstatieren die Autoren der europäischen Filmindustrie immer noch einen Mangel an Konkurrenz- 
fähigkeit mit den USA. Der Beitrag analysiert die Strategie und Effektivität des Programms und konstatiert, dass die im MEDIA Plus Programm vorgesehenen Maßnahmen eher kontraproduktiv wirken, da die strukturelle Fragmentierung der Filmproduktion in Europa erhalten bleibe.

Johnston, Russell: The Murray scheme: advertising and editorial independence in Canada, 1920. - S. 251-270

Cormack, Patricia: Angels, bells, television and Ireland: the place of the Angelus broadcast in the republic. - S. 271-288

\section{Jg 27 (2005) Nr 3}

Bergfelder, Tim: National, transnational or supranational cinema?: rethinking European Film Studies. - S. 315-332

Der Artikel diskutiert die Parameter und Vorstellungen, die die Forschung zum Europäischen Film seit 1990 bestimmt haben. Die wichtigste Feststellung ist dabei, dass der supranationale Charakter zumeist nicht berücksichtigt wurde, sondern die Perspektive der Diskurse eine nationale geblieben ist. Es wird angeregt, in Zukunft die verschiedenen Aspekte der europäischen Vernetzung in den Blick zu nehmen, bspw. Fragen des Multikulturalismus, der Koproduktionen oder der Aneignungsstrategien für ausländische Filme durch Übersetzung und Adaption.

Glevarec, Hervé: Youth radio as „social object“: the social meaning of „free radio“ shows for young people in France. - S. 333-352

Eine Besonderheit der französischen Radiolandschaft sind die sehr liberalen "Radio libre“-Sendungen der Radiosender, die auf ein junges Publikum ausgerichtet sind. Die Call-in-Sendungen stellen nach Ansicht der interviewten Jugendlichen (15-16 Jahre) Diskussionsraum für Probleme junger Leute zur Verfügung, zu Themen wie Sexualität und Beziehungen, Identität, Drogen und z. T. auch zu politischen Themen. Der Artikel weist auf die Besonderheit des doppelten Charakters des Mediums hin (z. B. in der Gleichzeitigkeit von Dialog und sozialer Interaktion) und die Metatextualität des Jugendradios. Die dem Artikel zugrundeliegende Studie nutzte eine neue Art der Rezeptionsanalyse, indem eruiert wurde, was die Programme für die Jugendlichen „repräsentieren“. So wurden vier Typen „sozialer Objekte“, die das Radio darstellen kann: Radio als „gemeinsamer Bereich“, als „QuasiInstitution“, als „soziales Ereignis“ und als „Mittel“.

Wilcox, Lynne: Metro, info, haro!: Fierce reactions to regime competition in the French newspaper industry. - S. 353-370

Mitra, Ananda: Creating immigrant identities in cybernetic space: examples from a non-resident Indian website. - S. 371-390

Sawhney, Harmeet: Arenas of innovation: understanding new configurational potentialities of communication technologies. - S. 391-414

Murray, Simone: Brand loyalties: rethinking content within global corporate media. S. $415-436$

\section{Media Perspektiven}

\section{(2005) Nr 3}

Zubayr, Camille: Tendenzen im Zuschauerverhalten: Fernsehgewohnheiten und Reichweiten im Jahr 2004. - S. 94-104

Krüger, Udo Michael: Das Bild der Migranten im WDR Fernsehen: Ergebnisse einer empirischen Programmanalyse. - S. 105-114

„Vor dem Hintergrund der politischen Debatten über Zuwanderung, multikulturelle Gesellschaft und demographische Entwicklung ist auch die Frage relevant, welchen Beitrag die Medien zum Gelingen des Integrationsprozesses leisten. Der öffentlich-rechtliche Rundfunk trägt dabei eine besondere Verantwortung. In der Vergangenheit wurde in Inhaltsanalysen von Fernsehprogrammen häufiger ein verzerrtes Bild von Migranten ermittelt. Eine Programmanalyse des Nonfictionangebots im WDR Fernsehen sollte klären helfen, wie es aktuell um das Bild der Migranten im öffentlich-rechtlichen Fernsehen bestellt ist. Basis war eine vierwöchige Stichprobe des Gesamtprogramms im Jahr 2003. Insgesamt behandelten danach rund 10 Prozent des gesamten Nonfictionangebots im WDR Fernsehen Migrationsthemen im weiteren Sinn. Besonders hoch war der Anteil in der tagesaktuellen regionalen Berichterstattung, der Themenkontext stammte oft aus dem Bereich Politik/Wirtschaft/Zeitgeist. Beiträge mit explizitem Bezug zu Migration und Migranten behandelten häufig auch soziale und kulturelle Themen. Es konnte dabei weder eine einseitig positive noch einseitig negative Tendenz in der Berichterstattung festgestellt werden. Zwei Drittel der Beiträge, in denen Akteure mit ausländischem Hintergrund auftraten, hatten keinen spezifischen Themenaspekt der Migration, sondern zeigten die Alltagsnormalität einer Gesellschaft, in der verschiedene Nationalitäten und Ethnien zusammenleben. Akteure mit Migrationshintergrund sind zumeist ,Durchschnittsbürger', in drei Viertel der Fälle kamen sie auch selbst zu Wort. Die Handlungsbereiche der Akteure waren zumeist der Alltag, Beruf und die Familie. Als Hauptergebnis wird festgehalten, dass die Umsetzung des Integrationsgedankens im WDR Fernsehen nicht in einer Sonderrubrik stattfindet, sondern in einer Vielzahl von Sendungen und Genres anzutreffen ist. Soziale Problemlagen werden dabei in einer Weise dargestellt, in der die Lebenssituation der Migranten möglichst authentisch und menschlich erscheint. Die Verstärkung negativer Stereotype wird vermieden, stattdessen Problemlagen nachvollziehbar gemacht und Nähe zu Personen hergestellt."

Gerhards, Maria: Die Offliner: eine homogene Gruppe der Internetverweigerer?: Zugangsbarrieren aus typologischer Perspektive. - S. 115124

„Unter den Offlinern, das heißt den Nicht-Nutzern des Internets, steigt zwar der soziale Anpassungsdruck, dennoch bleiben die Zugangsbarrieren bestehen. Dabei spielt das Alter der Menschen eine große 
Rolle: Während der Anteil der Jugendlichen (14 bis 19 Jahre), die zumindest gelegentlich online sind, laut ARD/ZDF-Online-Studie 2004 bei rund 95 Prozent liegt, sind es bei den ab 60-Jährigen nur 14,5 Prozent. Um die Offliner differenzierter betrachten zu können, wurde auf Basis der ARD/ZDF-Offline-Studie eine Offliner-Typologie gebildet, die fünf Offlinertypen unterscheidet. Die Spannweite reicht von den absolut Desinteressierten, eher Ablehnenden sowie Distanzierten, die sich (noch immer) nicht vorstellen können, welchen Nutzwert ihnen das Internet bieten könnte, über die Nutzungsplaner bis hin zu den Erfahrenen, die aber aktuell trotzdem nicht im Netz sind. Gegen die Anschaffung eines Internetanschlusses sprechen für die Ablehnenden die Kosten, technische Barrieren und mangelndes Interesse. Den Distanzierten fehlt der Nutzwert, sodass auch sie keinerlei Anschaffungsabsichten hegen. Die Nutzungsplaner, die sich vor allem unter den jüngeren Offlinern finden, spüren den sozialen Druck am stärksten, online zu gehen. Sie möchten den Anschluss an einen bestimmten gesellschaftlichen Standard nicht verlieren und nehmen das Internet in erster Linie als Informationsplattform wahr. Die Erfahrenen zeigen sich hingegen verhaltener, außer dass sie in höherem Maße wissen, welchen (persönlichen) Nutzwert das Internet aufweist. Immerhin eine Minderheit unter ihnen plant, sich einen Internetanschluss zuzulegen. Geht man davon aus, dass die Nutzungsplaner bereits überzeugt sind, gelten die Erfahrenen als nächster möglicher ,Schwellen-Typ'. Ihr vorhandenes Erfahrungswissen könnte sie - bei weiterer Senkung der Anschaffungskosten sowie positiver Verstärkung des Nutzwerts von Internet - schnell zu Nutzungsplanern werden lassen. Neben den Desinteressierten, überwiegend alte und weibliche Personen mit geringem Einkommen, die sehr starke Vorbehalte und Abneigungen gegenüber dem Internet aufweisen, sind die Ablehnenden und Distanzierten noch weit entfernt von einem direkten Kontakt mit dem Internet. Außer dem Abbau der technischen Barrieren durch eine leichtere Bedienung wären hier weitere, auf ihre Zielgruppe zugeschnittene Angebote, wie zum Beispiel Homebanking und bequemes Einkaufen im Netz, zu kommunizieren."

Colwell, Tim: Interaktives TV: als Ergänzung zum traditionellen Fernsehen akzeptiert: eine britische Studie zur Nutzung interaktiver Angebote. - S. 125-133

ARD-Forschungsdienst; Werbewirkung als Interaktion von Werbegestaltung und Rezipient. - S. 134-139

\section{(2005) Nr 4}

Matheus, Kirsten: Marktchancen von DAB und DVB-T: ein Lagebericht zu den digitalen terrestrischen Rundfunkübertragungstechnologien. - S. 142-151

„Der Artikel beschreibt die Marktzusammenhänge von Rundfunkverbreitungstechnologien im Allgemeinen und die Marktsituationen der Technologien DAB und DVB-T im Besonderen. Der Forschungsbericht entstand im Auftrag der Automobilindustrie, seine Befunde haben aber darüber hinaus allgemeine
Bedeutung. Bei DVB-T in Deutschland wurde der Markt durch die Kombination von Zwang durch $\mathrm{Ab}$ schaltung der analogen Sendeanlagen und gleichzeitigem Mehrwert durch Verdopplung der Programmanzahl (oder günstigerem Preis bei akzeptablem Angebot gegenüber Kabel) angeregt. DAB wurde in Großbritannien durch die Schaffung eines sichtbaren Mehrwerts - der Verdopplung der Programmanzahl ebenfalls erfolgreich am Markt eingeführt. Im Gegensatz zu DVB-T in Deutschland, wo die zusätzlichen, terrestrischen Programme bereits über Kabel und Satellit verbreitet wurden, wurden in Großbritannien auch viele Programme mit neuen Inhalten entwickelt, die nur über DAB zu empfangen sind. Bei DAB in Deutschland sieht die Situation anders aus. Die Sendeleistung ist zu gering, um die Masse erreichen zu können, DAB ist hierzulande stark auf den Empfang in - technisch aufwändigen und damit teuren - Autoradios ausgerichtet. Es gibt auch keine wirkliche Programmerweiterung, geschweige denn einen Zwang, die Technologie zu verwenden. Unter diesen Voraussetzungen kann sich die Technologie nicht durchsetzen; , digital' ist nichts, was einen Markt von alleine entstehen lässt. Nach Ansicht der Autoren fehlt es in Deutschland an einer kollektiven Motivation aller Beteiligten. Diese Motivation ist aber die Basis, aus der sich alle anderen Aktivitäten ergeben. Die Autoren listen eine Reihe von Aspekten auf, die auch in Deutschland die Digitalisierung des Hörfunks motivieren können. Die Automobilindustrie ist durchaus daran interessiert, eine Technologie wie DAB ins Fahrzeug zu bringen, da DAB neue Dienste und Hörfunkprogramme in besserer Qualität im Fahrzeug ermöglicht. Sie kann aber auf Marktentwicklungen bei DAB nur reagieren, nicht sie herbeiführen. Sie ist bei DAB nur einer von vielen Teilnehmern der Wirkungskette. Die Koordination dieser Teilnehmer ist notwendig - in der Motivation, in der Schaffung eines sichtbaren Mehrwerts und im weiteren Vorgehen. Wenn dieser Mehrwert vorhanden ist, wird der digitale Hörfunk auch in Deutschland erfolgreich sein."

Zubayr, Camille: Die Informationsqualität der Fernsehnachrichten aus Zuschauersicht: Ergebnisse einer Repräsentativbefragung zur Bewertung der Fernsehnachrichten. - S. 152-162

Feierabend, Sabine: Was Kinder sehen: eine Analyse der Fernsehnutzung Drei- bis 13Jähriger 2004. - S. 163-177

Blumers, Marianne: Fernsehprogramme und ihre Bewertung: das Programm-BewertungsVerfahren im SWR. - S. 178-183

„Die immer wiederkehrende Diskussion um Sinn und Nutzen der ,Quote im Fernsehen, um Programmstandards und Anpassung an den angeblichen Massengeschmack verweist nicht zuletzt auf das Spannungsverhältnis von Angebotsqualität und Zuschauerakzeptanz. Insbesondere für die öffentlich-rechtlichen Sender mit ihrem spezifischen Programmauftrag, der vor allem auch einen Qualitätsanspruch enthält, ist dieses Thema immer wieder aktuell. Vor dem Hintergrund eines intensivierten Wettbewerbs zwischen den Fernsehveranstaltern sind Instrumente gefragt, die sowohl für die programmstrategische Planung als auch für die Programmoptimierung auf der redaktionellen Ebene Kriterien und Daten für eine Bewertung des Programms liefern. Die Abteilung Medienforschung/ 
Programmstrategie im Südwestrundfunk (SWR) hat zu diesem Zweck (analog zu ähnlichen Initiativen in anderen öffentlich-rechtlichen Sendern) das Programm-Bewertungs-Verfahren (PBV) entwickelt. Dieses Instrument bringt so genannte Sendeplatzziele, das heißt von den jeweiligen Redaktionen formulierte Profile einzelner Sendungen, mit Zuschauerbewertungen für diesen Sendeplatz und Daten aus der regelmäßigen Zuschauerforschung in Zusammenhang. Das PBV leistet dabei dreierlei: eine Rückkopplung von Redaktionszielen mit Zuschauerwahrnehmungen, einen Beitrag zur Weiterentwicklung von Sendeplätzen und einen Input zur Entwicklung des Programmangebots insgesamt. In der Regel werden im Rahmen der PBV pro Sendungstest etwa 120 Personen gebeten, einen bestimmten Sendeplatz an vier aufeinander folgenden Terminen anzuschauen. Die Untersuchungsteilnehmer werden jeweils direkt nach einer Ausstrahlung telefonisch befragt und gebeten die Sendung anhand standardisierter Itemlisten und einer 4er-Skala („trifft voll und ganz zu“ bis „trifft gar nicht zu“) zu bewerten. Diese Ergebnisse können gegebenenfalls durch Gruppendiskussionen ergänzt werden. Die bisherigen Erfahrungen zeigen, dass das Programm-Bewertungs-Verfahren geeignet ist, Stärken und Schwächen einer Sendung herauszuarbeiten und konkrete Anhaltspunkte für eine Verbesserung zu liefern. Dies kann u. a. die Moderation einer Sendung, das Studio oder eventuelle Gesprächspartner betreffen. Vergleiche im Sinne eines Benchmarking können darüber hinaus mit Sendungen aus der selben Sparte, aber von anderen Sendern, durchgeführt werden und Hinweise für die Programmstrategie liefern. Im SWR ist es mit Hilfe des PBV möglich geworden, gezielt Verbesserungen an einzelnen Sendungen durchzuführen, die gleichzeitig eine bessere Beurteilung durch die Zuschauer und eine höhere Akzeptanz (gemessen an der Quote) bewirkten.“

\section{(2005) Nr 5}

Krüger, Udo Michael: Sparten, Sendungsformen und Inhalte im deutschen Fernsehangebot: Programmanalyse 2004 von ARD/Das Erste, ZDF, RTL, Sat.1, und ProSieben. - S. 190-204

Schütz, Walter J.: Deutsche Tagespresse 2004: Zeitungsmarkt trotz Krise insgesamt stabil. S. 205-232

Schütz, Walter J.: Redaktionelle und verlegerische Struktur der deutschen Tagespresse: Übersicht über den Stand 2004. - S. 233-242

Melischek, Gabriele: Der österreichische Zeitungsmarkt 2004: hoch konzentriert: Strukturen, Marktpotenziale, Anbieterkonzentration. -S. 243-252

\section{(2005) Nr 6}

Heffler, Michael; Pamela Möbus: Der Werbemarkt 2004: solides Wachstum nach dreijähriger Talsohle. - S. 258-266

„Das Werbejahr 2004 kann den klassischen Medien Anlass zur Zuversicht geben: Nicht nur die Brutto- werbeumsätze sind im vergangenen Jahr gestiegen, sondern auch bei den Nettoumsätzen konnte erstmals seit Jahren wieder ein Plus erzielt werden. Wachstumsmotor waren dabei die Handelsdiscounter, so Michael Heffler und Pamela Möbus in ihrem Rückblick, sie haben sich zur Branche mit dem höchsten Werbedruck im deutschen Markt entwickelt. Vom Werbewachstum profitierten die Werbeträger Tageszeitung und Radio am meisten."

Wild, Christoph: Synergien nutzen - mit Radio im Mediamix: Ergebnisse einer amerikanischen Studie zur Wirkung von Radiowerbung. S. $267-270$

„Radio erweist sich nach einer von Christoph Wild vorgestellten aktuellen Studie des US-amerikanischen Radio Ad Effectiveness Lab (RAEL) als ein wichtiges Medium zur Steigerung der Werbeeffizienz. Ein Media-Mix, der Radio mit einbezieht, erzielt mehr Werbewirkung als eine Mono-Kampagne mit TV oder Tageszeitungen. Radio, so die RAEL-Forscher, kann Personen ansprechen, die nicht von anderen Medien erreicht werden, und Radio kann Werbebotschaften anderer Medien verstärken.“

Blödorn, Sascha, Maria Gerhards: Veränderungen der Medienzuwendung mit dem Älterwerden: Daten zur Nutzung elektronischer Medien 2004. - S. 271- 283

"Wie sich die Nutzung elektronischer Medien mit "dem Älterwerden verändert, analysieren Sascha Blödorn und Maria Gerhards. Rund 63 Prozent der Bevölkerung ab 14 Jahre sind mittlerweile über 40 Jahre alt. Das Alterwerden beeinflusst die Mediennutzung, wobei sich zwischen den einzelnen Alterssegmenten deutliche Unterschiede zeigen. Die stärksten Veränderungen werden durch den Eintritt in die Rentenund Pensionsphase markiert, also bei den 60- bis 69Jährigen. Generell steigt die Fernsehnutzung mit zunehmendem Alter an, beim Radio ist es umgekehrt. Die 40- bis 59-Jährigen werden vom Hörfunk noch überdurchschnittlich gut erreicht. Unter den ab 60Jährigen sind Onlinenutzer nach wie vor relativ selten, während ihr Anteil bei den 50- bis 59-Jährigen mittlerweile fast den Durchschnittswert der Bevölkerung erreicht hat."

Frees, Beate; Martin Fisch, Alexander Ebbes: „Usertacking“: Nutzungspfade im Webangebot: Anwendung eines Instruments der Onlineforschung im Rundfunkbereich. - S. 284-289

„Damit Fernsehsender ihre programmbegleitenden Onlineangebote den Gewohnheiten und Bedürfnissen der User entsprechend optimieren können, müssen möglichst detaillierte und aktuelle Kenntnisse des Nutzerverhaltens vorliegen. Beate Frees, Martin Fisch und Alexander Ebbes berichten über ein so genanntes Usertracking-Verfahren des ZDF, mit dessen Hilfe den Redaktionen wichtige Kennzahlen zur Verbesserung des Webangebotes an die Hand gegeben werden sollen." 


\section{Media Perspektiven, Dokumentation}

\section{(2005) Nr 1}

Rundfunkrechtliche Staatsverträge in der Fassung des Achten Rundfunkänderungsstaatsvertrages (in Kraft seit 1. April 2005). - S. 1-80

\section{Media psychology}

\section{Jg 7 (2005) Nr 2}

Konijn, Elly A.: Some like it bad: testing a model for perceiving and experiencing fictional characters. - S. 107-144

„We developed an encompassing theory that explains how readers of fiction and spectators of motion pictures establish affective relationships with fictional characters (FCs). The perceiving and experiencing fictional characters (PEFiC) theory is anchored in art perception, psychological aesthetics, and social and emotion psychology and addresses both the complexity and intrinsic affectivity involved in media exposure. In a between-subject design $(\mathrm{N}=312)$, engagement and appreciation were measured as a function of the ethics (good vs. bad), aesthetics (beautiful vs. ugly), and epistemics (realistic vs. unrealistic) of eight protagonists in feature movies. The PEFiC model best fit the data with a unipolarity of factors and outperformed traditional theories (identification, empathy): The trade-off between involvement and distance explained the appreciation of FCs better than either distance or involvement alone. The mediators similarity, relevance, and valence exerted significant (interaction) effects, thus complicating the results. Furthermore, the effects of mediated bad persons differed strongly from ethically good ones."

Raney, Arthur A.: Punishing media criminals and moral judgment: the impact on enjoyment. -S. 145-164

„In this study, I investigated the relation between moral judgment and the enjoyment of crime dramas by varying the relative severity of punishment levied for a crime. One hundred fifty-one participants rated their enjoyment of a video clip depicting a crime with the perpetrator being punished either excessively or not at all. In keeping with previous literature, I predicted that the different punishments would elicit different levels of moral judgment, which would then impact enjoyment. The results indicate that crime drama enjoyment was consistently predicted by certain social justice attitudes and resulting moral judgments about the content. The findings lend support to moral sanction theory and the integrated model of crime drama enjoyment and shed further insight into how viewer cognitions impact dispositional affiliations formed toward characters in media entertainment."

Trepte, Sabine: Daily Talks as self-realization: an empirical study on participation in daily talks shows. - S. $165-190$

"In this study, I examined the reasons why television audiences want to be on television and why they want to participate in shows as talking guests. To answer these questions, I assessed German daily talk shows and their audiences. The theory of symbolic self-completion (Wicklund \& Gollwitzer, 1982) was applied to explain the wish to participate in a show. Self-realization is suggested as a higher order motive, including goals such as getting therapy and confessing in such a show. Two empirical studies were conducted. In the first study, 66 viewers of daily talk shows were interviewed with a qualitative procedure to explore if and why they would attend a talk show. Clues to lay participation as an act of self-symbolizing were deduced. Furthermore, the results of the study show that viewers engaged one of two viewing modes, distanced or involved. A follow-up experiment with 33 research participants was carried out. The findings support the hypothesis that people who are considering media participation strive for self-realization and self-symbolizing."

Bracken, Cheryl Campanella: Presence and Image Quality: the case of High-Definition Television. - S. 191-206

„Previous research has demonstrated that form variables can increase television viewers' sense of presence. The current broadcasting of high-definition television (HDTV) programming makes testing this relation between form and presence possible in a new context, image quality. In this experiment, television viewers watched either HDTV or standard-definition television images and then rated their viewing experience on a pencil-and-paper questionnaire. The results demonstrate that HDTV provided viewers with a greater sense of presence than the current standard, thus providing empirical evidence for the claim that improved image quality will lead to television viewers' experiencing presence. Additionally, the results suggest that with the coming of HDTV, sensations of presence will be both stronger and more common."

Hoffner, Cynthia A.: Enjoyment of mediated fright and violence: a meta-analysis. - S. 207235

„In this meta-analysis, we synthesized data from published journal articles that investigated viewers' enjoyment of fright and violence. Given the limited research on this topic, this analysis was primarily a way of summarizing the current state of knowledge and developing directions for future research. The studies selected (a) examined frightening or violent media content; (b) used self-report measures of enjoyment or preference for such content (the dependent variable); and (c) included independent variables that were given theoretical consideration in the literature. The independent variables examined were negative affect and arousal during viewing, empathy, sensation seeking, aggressiveness, and the respondents' gender and age. The analysis confirmed that male viewers, individuals lower in empathy, and those higher in sensation seeking and aggressiveness reported more enjoyment of fright and violence. Some support emerged for Zillmann's (1980, 1996) model of suspense enjoyment. Overall, the results demonstrate the importance of considering how viewers interpret or appraise their reactions to fright and violence. However, the studies were so diverse in design and measurement methods that it was difficult to identify the underlying processes. Suggestions are proposed for future research that will move toward the integration of separate lines of inquiry in a unified approach to understanding entertainment." 


\section{medien + erziehung}

\section{Jg 49 (2005) Nr 2}

Kübler, Hans-Dieter: Computer installiert (Medien-) Pädagogik passé?. - S. 9-16

„Die Ausstattung deutscher Schulen mit Computern und Internetanschlüssen hat sich in den letzten Jahren deutlich verbessert. Doch haben sich damit auch die Erwartungen bezüglich neuer Lernformen und medienpädagogischer Erfolge erfüllt, die damit verbunden waren? Dieser Beitrag untersucht, wie es um die Entwicklung der Medienpädagogik und -kompetenz derzeit bestellt ist."

Spanhel, Dieter: Zehn Jahre schulische Medienpädagogik. - S. 17-22

Zehn Jahre nach der Veröffentlichung des Orientierungsrahmens "Medienerziehung in der Schule" der Bund-Länder-Kommission für Bildungsplanung und Forschung zieht Dieter Spanhel kritisch Bilanz: „Die empirische Ergebnisse zeigen, dass im Verlaufe der letzten zehn Jahre weder eine nachhaltige Intensivierung der Medienerziehung noch eine deutliche Verbesserung der Lehr-/Lernprozesse durch den Einsatz der neuen Medien erreicht werden konnte. Eine auf medienpädagogischen Maßnahmen gegründete Schulentwicklung lässt sich erst recht nicht beobachten." (S. 20) Schulische Medienpädagogik müsste sich aus seiner Sicht aktiver und intensiver als bisher an interner Schulentwicklung beteiligen. Zudem sollte der Medienbildung - der kritischen Auseinandersetzung mit den Medien und Ausbildung einer „moralischen Verantwortungshaltung" (S. 21) - mehr Raum beigemessen werden. Durch die Einrichtung von Ganztagsschulen bieten sich diesbezüglich der schulischen Medienpädagogik neue Entfaltungs- und Gestaltungsmöglichkeiten.

Fritsche, Christian: Qualitätskriterien für Medienprojekte. - S. 23-27

Ostermann, Sandra: Offene und interdisziplinäre Lernkultur mit Digitalen Medien: das Projekt ZIM@School.-S. 28-33

„In dem vorliegenden Beitrag werden Ergebnisse aus dem ersten Jahr (2003/04) des Lehr- und Forschungsprojekts ZIM@School (ZIM heißt „Zentrum für Interaktion mit Digitalen Medien") vorgestellt, das von der Cornelsen-Stiftung "Lehren und Lernen“" gefördert und in der Arbeitsgruppe „Digitale Medien in der Bildung" (DiMeB) and er Universität Bremen in Kooperation mit Bremer Schulen durchgeführt wird. Neben Ausgangssituation und Beschreibung des Projekts werden die zentralen Aspekte der wissenschaftlichen Begleitforschung auf der Grundlage von teilnehmenden Beobachtungen und qualitativ erhobenen Daten genannt."

Anfang, Günther et al: Medienpädagogische Projekte an Schulen. - S. 34-42

Der Beitrag umfasst Kurzdarstellungen von verschiedenen medienpädagogischen Praxisprojekten.

Schiffer, Sabine: Der Islam der Medien. S. 43-48

Die Autorin beschreibt am Beispiel der Darstellung des Islams das meinungsbildende Potenzial der medialen Berichterstattung. An verschiedenen Beispielen aus dem Fernsehen und der Presse illustriert sie, auf welche Weise Vorurteile und Stereotype durch die Medien gefestigt bzw. geschürt werden.

Mikos, Lothar: Selbstermächtigung mit populären Fernsehformaten. - S. 54-59

Pitum, Sandra: Geschlechtsspezifische Rezeptionswirkung von Spielfilm-Trailern. -S. 60-64 „Trailer sind heutzutage wesentliche Bestandteile des Fernsehprogramms; sie dienen im Kampf um Einschaltquoten der Eigenwerbung und Imagebildung. Aus diesem Grund ist es notwendiger denn je geworden, die Trailer anhand sorgfältig durchdachter Konzepte zu produzieren. In der diesem Aufsatz zugrunde liegenden Studie wurde die Rezeption und Wirkung von Spielfilm-Trailern näher untersucht, und zwar unter geschlechtsspezifischem Aspekt. Als wesentliche Elemente des Trailers wurden hierbei Bildaufbau und Musikuntermalung variiert und auf Erinnerung, Bewertung, Sehabsicht und emotionales Medienerleben hingetestet."

\section{Jg 49 (2005) Nr 3}

Tully, Claus J.: Handys und jugendliche Alltagswelt. - S. 11-16

„Inzwischen verfügen rund 90 Prozent der Jugendlichen über ein Handy. Als Kommunikations- und Medienzentrale strukturiert es den mobilen Alltag und dient als Werkzeug zur Gestaltung und Organisation der Peergroup. Gleichzeitig trägt es durch individualisierte Nutzung zur Identitätsfindung bei.“

Schulz, Iren: Zwischen Reiz und Risiko: Jugendliche über Handys und Mobilfunkangebote. - S. $17-23$

Der Beitrag gibt einen Einblick über die Rolle von Mobilfunkkommunikation im Alltag von Jugendlichen und lässt dabei vier Jugendliche (14 bis 16 Jahre), die im Rahmen eines großangelegten Monitorings zum Thema Medienkonvergenz (www.medienkonvergenz-monitoring.de) interviewt wurden, zu Wort kommen. Die Aussagen der Jugendlichen bestätigen Ergebnisse anderer Studien zur Mobilfunkkommunikation, verweisen aber auf anschauliche Weise auf die individuellen Unterschiede. Zusammenfassend resümiert die Autorin: „Ingesamt bleibt festzuhalten, dass sowohl forschungs- als auch medienpädagogischer Handlungsbedarf besteht, nicht nur was den Umgang Heranwachsender mit Handy und Mobilfunkinhalten, sondern auch den Umgang mit konvergenten, im Medienverbund vermarkteten Medienangeboten betrifft."

Selmer, Lena: „Nicht nah, aber immer für dich da!": Erreichbarkeit im Familienalltag. S. 24-27

„Der Aufsatz befasst sich mit typischen Funktionen und Formen der Handynutzung von Familien mit im Haushalt lebenden Kindern. Am Beispiel Familienalltag soll im Folgenden aufgezeigt werden, welche Prozesse sich durch gegenseitige "Connectivity" (Tomlinson 1999) ergeben, d. h. einerseits durch eigenen Erreichbarkeit und andererseits durch die Möglich- 
keit, mit abwesenden Personen in Kontakt zu treten. Der Gebrauch eines Mobiltelefons eröffnet zahlreiche Chancen zur gegenseitigen Abstimmung, birgt aber auch Probleme und Risiken.“

Döring, Nicola: Handy und SMS im Alltag: Ergebnisse einer Befragungsstudie. - S. 29-34

„Welche Bedeutung haben das Handy und insbesondere der SMS-Dienst für Kinder und Jugendliche? 400 Schülerinnen und Schüler wurden zu den wichtigsten Aspekten ihre Handy- und SMS-Nutzung schriftlich befragt: Nutzungsintensität und Finanzierung, Nutzungsformen, Kommunikationspartner, SMS-Anlässe und SMS-Sprache."

Ehler, Karin: Mittler zwischen den Generationen?: das Handy im Fokus des Kinder- und Jugendschutzes. - S. 35-39

„Die Landesstelle Kinder- und Jugendschutz Sachsen-Anhalt e.V. bietet Beratungen, Informationsveranstaltungen, Fortbildungen und Workshops zu Themen an, die im Zusammenhang mit dem Kinder- und Jugendschutzgesetz (JuSchG) oder dem Jugendmedienschutzstaatsvertrag (JMStV) relevant sind, etwa im Bereich Computerspiele, Filme, aber eben auch Handy. Für merz führte Karin Ehler ein Interview mit dem Geschäftsführer der Landesstelle, Arnfried Böker, über die jugendschutzrelevanten Aspekte des Handygebrauchs."

Kistner, Hans Peter: Jugendliche „reagieren gut auf Marketing“. - S. 40-41

Schuster, Katrin: „Big Brother“ forever: Anmerkungen zur sechsten Staffel des RealityFormats. - S. 45-49

Schäfer, Mike Steffen: Jugendliche Fans und ihr Medienumgang. - S. 49-53

Hoffmann, Bernward: „Kino macht Schule“: Filme als schulisches Lernmedium. - S. 50-55

Lange, Andreas: Emotionen als Wirtschaftsgut: Herausforderung an eine reflexive Medienpädagogik. - S. 56-59

Kern, Gregor: Behinderung, Medien und Schule: das Projekt „Objektiv“: ein Stück Lebensqualität vermitteln. - S. 60-65

Vorgestellt wird das ambitionierte Projekt OBJEKTIV, das sich seit Ende der 90er Jahre bemüht, über Filmpräsentationen und -gespräche einen kommunikativen Austausch zwischen Menschen mit und ohne Behinderung herzustellen. Das Angebot von OBJEKTIV richtet sich vor allem an Schulen. Über die bereitgestellten Filme wird eine gemeinsame Gesprächsgrundlage geschaffen, die es den Teilnehmenden erleichtert, über das Thema Behinderung offen zu kommunizieren. Die Filmgespräche werden zudem von Personen mit Behinderung moderiert und bieten auf diese Weise eine besondere Form der Auseinandersetzung.

\section{Medien \& Zeit}

\section{Jg 20 (2005) Nr 2}

Fickers, Andreas: Das „European Television History Network“: europäische Fernsehgeschichtsschreibung in vergleichender Perspektive. - S. 4-11

Desmet, Lieve: Celebrating 50 years of TV on the Flemish Public Broadcaster, VTR in Belgium: entertaining and building a collective memory. - S. 12-19

Baungartner, Susanna: Frauenbewegtes Fernsehen im ORF der 1970er: medienhistorische und genderperspektivische Betrachtungen zur ORF-Magazinsendung „Prisma“ unter der Leitung von Trautl Brandstaller. - S. 20-29

Lietz, Thomas: Fernsehnutzung in der DDR als kommunikationshistorisches Problem: $\mathrm{Me}-$ thodologie und Quellen. - S. 30-43

\section{Medien Journal}

\section{Jg 29 (2005) Nr 1}

Scheer, Uta: Männerzeitschriften - Frauenzeitschriften: Systematisierung eines gemeinsamen Forschungsfeldes. - S. 23-34

Scheer, Uta: Vom Nutzen notorisch professioneller Beobachtungen. - S. 35-44

Flicker, Eva: Männlichkeitsformen in Outdoor-Reality-TV-Shows am Beispiel von „Expedition Österreich“. - S. 45-57

\section{Medien Wirtschaft}

\section{Jg 2 (2005) Nr 2}

Dietl, Helmut: Piraterie auf dem Tonträgermarkt und die Evolution von neuen Geschäftsmodellen in der Musikproduktion. - S. 53-62

Rottenbiller, Silvia: Voice over IP: Grundlagen und Besonderheiten. - S. 63-67

\section{Multimedia und Recht}

\section{Jg 8 (2005) Nr 4}

Altenburg, Stephan: Telekommunikation am Arbeitsplatz. - S. 135-139

Vander, Sascha: Eingriffe in das allgemeine Fernsabsatzrecht: Gesetz zur Änderung der Vorschriften über Fernabsatzverträge bei Finanzdienstleistungen. - S. 139-145 
Berberich, Matthias: Die urheberrechtliche Zulässigkeit von Thumbnails bei der Suche nach Bildern im Internet. - S. 145-148

„Bei einer Suche nach Bildern im Internet ist im Gegensatz zur Suche nach Text für die Anzeige der Ergebnisse eine verkleinerte Vorschau kompletter Bilder nötig. Wenn diese Werkcharakter aufweisen, ist das als öffentliche Zugänglichmachung ein Eingriff in die dem Urheber vorbehaltenen Verwertungsrechte. Dieser Beitrag soll zeigen, daß einerseits die potentielle Verwertung durch Zustimmung des Urhebers an zu hohen Transaktionskosten wirtschaftlich scheitern, andererseits die zustimmungsfreie Nutzung die Interessen des Urhebers nicht beeinträchtigen würde. Das Verbotsrecht führte allein zur Behinderung auf dem nachgelagerten Angebotsmarkt für Suchdienste nach Bildern im Internet, was letztlich weder dem Urheber noch der Suchdienste nachfragenden Allgemeinheit dient und einer effizienten Faktorallokation entgegenläuft. Ein gangbarer rechtlicher Ansatz zur Korrektur liegt in der Annahme einer konkludenten Einwilligung des Urhebers, deren Umfang und Grenzen aufgezeigt werden."

Arlt, Christian: Ansprüche des Rechteinhabers bei Umgehung seiner technischen Schutzmaßnahmen. - S. 148-154

Hermerschmidt, Sven: Adresshandel durch die GEZ?: zur Befugnis der GEZ, Anschriften beim kommerziellen Adresshandel zu erheben. -S. $155-160$

„Die Finanzierung des öffentlich-rechtlichen Rundfunks ist seit Jahren immer wieder Gegenstand der öffentlichen Diskussion. Neben der Frage nach der Höhe der Rundfunkgebühr stehen regelmäßig auch die Methoden der öffentlich-rechtlichen Rundfunkanstalten und der von ihr beauftragten Gebühreneinzugszentrale (GEZ) bei der praktischen Durchführung des Gebühreneinzugs im Fokus der Öffentlichkeit. Dies betrifft sowohl den Umgang mit den Gebührenzahlern als auch die Art und Weise der Verarbeitung personenbezogener Daten. Zur Ermittlung tatsächlicher oder vermeintlicher Schwarzseher und -hörer bedienen sich die Rundfunkanstalten zunehmend des kommerziellen Adresshandels, um nach bestimmten Kriterien gezielt selektierte Adressdaten von mehreren Millionen Menschen im Jahr zu erhalten. Gerade diese Praxis wurde und wird von den Betroffenen, aber auch in der Öffentlichkeit kritisch betrachtet. Im folgenden Beitrag soll daher geprüft werden, ob diese Praxis der GEZ aus datenschutzrechtlicher Sicht zulässig ist. Dabei wird in erster Linie auf die für den Rundfunk Berlin-Brandenburg (RBB) geltende Rechtslage abgestellt. Die Aussagen lassen sich jedoch überwiegend auf die übrigen Landesrundfunkanstalten übertragen."

Jochum, Heike: Steht die Zugangsregulierung im Ermessen der Regulierungsbehörde für Telekommunikation und Post?. - S. 161-165

„Das neue Telekommunikationsgesetz (TKG) hat die tk-rechtliche Marktregulierung grundlegend verändert. Der zweite Abschnitt des neuen TKG statuiert ein neuartiges Konzept tk-rechtlicher Zugangsregulierung. Dabei enthält dieses Regelwerk eine Vielzahl sog. ,Kann-Vorschriften‘. Das neue TKG scheint damit die Zugangsregulierung ins pflichtgemäße Ermes- sen der Regulierungsbehörde für Telekommunikation und Post (Reg TP) zu stellen. Die Analyse der Entstehungsgeschichte, der Regelungssystematik sowie der europarechtlichen Grundlagen der Normen und ihres Telos zeigt jedoch, daß die Ermessensspielräume der Reg TP im Bereich der tk-rechtlichen Zugangsregulierung weitaus geringer sind, als es auf den ersten Blick scheint."

Bröcher, Julia: Domainnamen und das Prioritätsprinzip im Kennzeichenrecht. - S. 203-208

Schmitz, Florian: Die Haftung kommerzieller Meinungsportale im Internet. - S. 208-212

„Der Einfluss von Meinungsportalen auf den Absatz eines Produkts ist nicht zu unterschätzen. Immer öfter werden sie neben unabhängigen Produkttests zu Rate gezogen. Zwangsläufig stellt sich die Frage, ob und wem gegenüber sich Unternehmen gegen negative Bewertungen ihrer Produkte in kommerziellen Meinungsforen wehren können. Auf der anderen Seite ist es für Betreiber eines Meinungsportals von existenzieller Bedeutung, ob sie für Außerungen ihrer Nutzer haftbar sind. In Betracht kommen insbesondere Unterlassungs- und Schadensersatzansprüche. Diese scheitern jedoch häufig bereits daran, dass es sich bei Produktbewertungen regelmäßig um Werturteile handelt. Sollte dennoch einmal eine Äußerung rechtsverletzender Natur sein, bestehen gegen den Betreiber erst dann Ansprüche, wenn er von der rechtswidrigen Äußerung Kenntnis erlangt hat. Die Durchsetzung von Ansprüchen gegenüber dem Verfasser scheitert in der Praxis häufig an der Anonymität der Bewertung. Der Weg über eine Offenlegung der Nutzerdaten durch den Portalbetreiber bleibt dem Betroffenen regelmäßig mangels eines Auskunftsanspruchs und teilweise wegen datenschutzrechtlicher Erwägungen versperrt."

Sujecki, Bartosz: Erste Überlegungen zum europäischen elektronischen Mahnverfahren. S. 213-216

Hoenike, Mark: Die Besicherung von Krediten an Telekommunikationsunternehmen: eine Bestandsaufnahme unter der Geltung des neuen TKG. - S. 217-222

\section{Jg 8 (2005) Nr 5}

Rossnagel, Alexander: Das europäische Medienrecht. - S. 271-279

„Die medienrechtliche Diskussion in Deutschland, aber auch in anderen Mitgliedstaaten, hat sich bisher (zu) wenig mit den Maßgaben des europäischen Medienrechts befasst und wurde (zu) häufig von aktuellen Themen auf nationaler Ebene überlagert. Dies wird dem großen Einfluss, den das Gemeinschaftsrecht auf die Ausgestaltung der nationalen Medienordnungen ausübt und künftig in (wohl) zunehmendem Maß ausüben wird, nicht gerecht, In den letzten 25 Jahren hat sich ein eigenständiges, europäisches

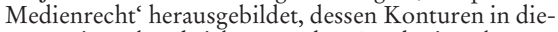
sem Beitrag beschrieben werden. Nach einer kurzen Einführung (I.) werden ausgehend von den Grundrechten des Europäischen Verfassungsvertrags und Grundfreiheiten des EGV (II.) die wichtigsten Regelungsbereiche des europäischen Medienrechts skiz- 
ziert und deren Entwicklungstendenzen angedeutet (III.). Ein Ausblick schließt den Beitrag ab (IV.).“

Thaenert, Wolfgang: Der Einfluss der EU-Medienpolitik auf die nationale Rundfunkordnung. - S. 279-284

„Die Europäische Union versucht, der ,grenzüberschreitenden' Dimension des Rundfunks durch den Aufbau einer europäischen Medienordnung gerecht zu werden. Dabei ist es nicht einfach, den strukturellen Anforderungen der Materie Herr zu werden. Die ineinander greifenden technischen, ökonomischen und rundfunkrechtlichen Aspekte führen $\mathrm{zu}$ einer komplexen Interessenlage. Die überwiegend wirtschaftlich motivierte Errichtung eines gemeinsamen Marktes ohne Hindernisse steht dabei der nach deutschem Recht zu wahrenden Funktion des Rundfunks für die Meinungsbildung in einer demokratischen Gesellschaft gegenüber. Aus der Vielzahl der Maßnahmen und Rechtsinstrumente der Europäischen Union und deren Einflüssen auf die deutsche Rundfunkordnung greift der [...] Beitrag aus Platzgründen hier nur einige gleichsam pars pro toto heraus." Hierzu zählen die EG-Fernsehrichtlinie und der neue gemeinsame Rechtsrahmen für elektronische Kommunikationsnetze und -dienste sowie die Einflüsse des Europäischen Wettbewerbsrechts auf die Rundfunkgebühr und deren Verwendung.

Nichel, Eva-Maria: Konvergenz der Medien: Auswirkungen auf das Amsterdamer Protokoll und das Europäische Beihilferecht. -S. 284-288

Der Beitrag beleuchtet die Erscheinungsformen der Konvergenz und Veränderungen im Rezeptionsverhalten, die Ausstrahlungswirkungen des Beihilferechts und die Bedeutung des Amsterdamer Protokolls. Die Autorin geht davon aus, dass dem Amsterdamer Protokoll ein funktionaler Rundfunkbegriff zugrunde liege, der auch Onlineangebote erfasse.

Kümmel, Annette: Gewährleisten die Regionalprogramme die Vielfaltssicherung?: eine kritische Bestandsaufnahme zwischen Vielfaltssicherung, Standortpolitik und europarechtlichen Vorgaben. - S. 288-291

In den Programmen von Sat.1 und RTL werden Regionalfenster veranstaltet bzw. ausgestrahlt. Der Beitrag zieht aus Sicht der ProSiebenSat.1 Media AG Bilanz und hinterfragt, inwieweit die ursprünglich verfolgten Zielsetzungen im Zeitalter der Digitalisierung aller Übertragungswege noch Gültigkeit besitzen und ob die Regionalfenster in der heute gesetzlich vorgeschriebenen Form geeignet sind, diese Ziele zu erreichen.

Christmann, Sabine: Der Markt für Breitbandkabel in der digitalen Übergangsphase: ordnungspolitische Herausforderungen für die deutsche Medienpolitik. - S. 291-294

„Die Entwicklung der Rundfunkmärkte stellt die Ordnungspolitik vor große gesetzgeberische Herausforderungen. Die Rahmenbedingungen bei der Umstellung von analoger auf digitale Übertragung definieren zugleich die wettbewerblichen Ausgangspositionen der Marktteilnehmer in einer digitalisierten Welt. Dies gilt insbesondere für das Breitbandkabel als wichtigsten Übertragungsweg für Rundfunkanbieter."

Schächter, Markus: Europa-Berichterstattung im ZDF: Vernetzung als Beitrag zur Entstehung einer „Europäischen Öffentlichkeit“. S. $294-298$

\section{Jg 8 (2005) Nr 6}

Schmidl, Michael: E-Mail-Filter am Arbeitsplatz. - S. 343-348

Kaufmann, Noogie C.: Metatagging: Markenrecht oder reformiertes UWG?. - S. 348-353

Attendorn, Thorsten: Von Gleichheit, Gleichwertigkeit und Chancengleichheit: eine Skizze des neuen tk-rechtlichen Gleichbehandlungsgebots. - S. 353-358

Der Beitrag stellt eine Skizze des neuen tk-rechtlichen Gleichbehandlungsgebots in $\$ 19$ TKG. „Die Regulierungspraxis hat gezeigt, dass gerade das Verbot, als Marktbeherrscher Wettbewerber zu diskriminieren, für reichlich Streitstoff zwischen den betroffenen Unternehmen ebenso sorgt wie für Uneinigkeit in der Rechtsprechung. Gegenüber den bisherigen Varianten des Diskriminierungsgebots ist das neue Gleichbehandlungsgebot sowohl seinem Gegenstand als auch seinem Inhalt nach weiter gefasst: Es gilt nunmehr für Vereinbarungen über jegliche Form des Zugangs und enthält insbesondere das bislang vielfach vermisste Verbot unbilliger Behinderung. Da der Kern der Regelung aus unbestimmten Rechtsbegriffen besteht und sich nur teilweise auf tk- oder wettbewerbsrechtlich vertrautem Terrain bewegt, ist [...] das Maß an Rechtsklarheit nicht in gleicher Weise gewachsen. Dieser Aufsatz stellt die Grundzüge des neuen Gleichbehandlungsgebots dar und soll zur Entwicklung von Rechtsklarheit beitragen."

Hamm, Axel: Geldausgleich für Wegerechte zum Aufbau öffentlich genutzter Telekommunikationsnetze. - S. 358-364

\section{Multimedia und Recht, Beilage}

\section{Jg 8 (2005) Nr 5}

Schuster, Fabian et al: Entwicklung des Internet- und Multimediarechts im Jahre 2004. S. 1-37

\section{Jg 8 (2005) Nr 6}

Konsistenzgebot und Entgeltregulierung: Workshop am 29.11.204, RegTP. - S. 1-36

New media \& society

Jg 7 (2005) Nr 2

Chalaby, Jean K.: Deconstructing the transna- 
tional: a typology of cross-border television channels in Europe. - S. 155-176

Es werden vier Typen von transnationalen Fernsehkanälen unterschieden: ethnische, gebietsübergreifende und paneuropäische Kanäle sowie paneuropäische Netzwerke. Auf der Basis von 40 Interviews mit Führungskräften soll gezeigt werden, wie grenzüberschreitende Programme mit Nationalstaaten, geographischem Raum und Kultur zusammenhängen.

Coleman, Stephen: New mediation and direct representation: reconceptualizing representation in the digital age. - S. 177-198

Wyatt, Sally et al: The digital divide, health Information and everyday life. - S. 199-218

Der Beitrag präsentiert eine Studie zur Nutzung des Internet zu den Themen Menopause und Hormonersatztherapie bzw. erektile Dysfunktion und Viagra und diskutiert vor diesem Hintergrund das Konzept des Digital Divide.

Richardson, Margaret: „Getting on“: older New Zealanders' perceptions of computing. S. 219-246

Wiklund, Hans: A Habermasian analysis of the deliberative democratic potential of ICT-enabled services in Swedish municipalities. S. $247-270$

\section{Jg 7 (2005) Nr 3}

Salter, Lee: Colonization tendencies in the development of the world wide web. - S. 291-310

Dijck, José van: From Shoebox to performative agent: the Computer as personal memory machine. - S. 311-332

Jansz, Jeroen: Gaming at a LAN event: the social context of playing video games. - S. 333356

Chan, Joey Ka-Ching: Lifestyles, reliance and traditional news media, and online news adoption. - S. 357-382

Valkenburg, Patti M.: Adolescents' identity experiments on the Internet. - S. 383-402

Röhle, Theo: Power, reason, closure: critical perspectives on new media theory. - S. 403-422

\section{Nordicom Review}

\section{Jg 26 (2005) Nr 1}

Bruhn Jensen, Klaus: Interactivity in the Wild: an Empirical Study of „Interactivity“ as Understood in Organizational Practices. - S. 3-30 Ridell, Seija: Mediating the Web as a Public Space: a Local Experiment in the Creation of

\section{Online Civic Genres. - S. 31-48}

Ottosen, Rune: The Norwegian Media Image of the War in Afghanistan: Peacekeeping or Agression?. - S. 95-110

Gynnild, Astrid: Winner Takes It All: Freelance Journalism on the Global Communication Market. - S. 111-120

Büttner, Christian: The Protection of Minors Against Harmful Media Content in Europa: How European Film Classifiers View Childhood and Adolescence. - S. 121-130

Olsson, Tobias: Young Citizens, ICTs and Learning: A Design for a Study of the Media and Political Activity. - S. 131-140

Hurme, Pertti: From Division to Cooperation: the Finnish University Network for Communication Sciences. - S. 141-144

Fornäs, Johan: The Advanced Cultural Studies Institute of Sweden / ACSIS: a National Centre for Transnational and Interdisciplinary Cultural Research. - S. 145-150

\section{Political communication}

\section{Jg 22 (2005) Nr 2}

Dahlgren, Peter: The Internet, Public Spheres, and Political Communication: Dispersion and Deliberation. - S. 147-162

Ausgehend von der Feststellung einer Destabilisierung der politischen Kommunikation diskutiert der Autor die Rolle von Internet und Öffentlichkeit bei der Informationsvermittlung und Deliberation. Dem Internet werden positive Effekte und Einschränkungen zugeschrieben. Das Internet kann die Öffentlichkeit erweitern, pluralisieren und Deliberation erleichtern. Allerdings entsprechen nicht alle Diskussionen im Internet den deliberativen Idealen. Dahlgren sieht sein Konzept der „civic cultures“ als zusätzlichen Weg, um die Bedeutung von politischer Partizipation via Internet zu untersuchen. Online-Nutzer werden hier in ihrer Rolle als Bürger betrachtet.

Muhlberger, Peter: Human Agency and the Revitalization of the Public Sphere. - S. 163-178

„This article introduces a theory of human agency that helps identify and integrate factors that may be important for explaining why the advent of the Internet has not revitalized the public sphere. The theory suggests three types of interrelated explanations. One type of explanation focuses on the economy of attention limits to human information processing and resulting tradeoffs. A second type of explanation, psychosocial structure, includes the development of routines of Internet use that confine that use to conventional activity. It also includes the decline of traditional political organization, which reduces participation incentives. Ultimately, however, attention and structure serve only as deterrents, not as bars to 
revitalization. Political disinterest serves as the final bar. This disinterest can best be understood as due to developmental factors that serve as a crucial impediment to a flourishing public sphere.“

Cammaerts, Bart: Online Political Debate, Unbounded Citizenship. and the Problematic Nature of a Transnational Public Sphere. - S. 179196

„Citizenship has always been a dynamic notion, subject to change and permanent struggle over its precise content and meaning. Recent technological, economic, and political transformations have led to the development of alternative notions of citizenship that go beyond the classic understanding of its relationship to nation states and rights. Civil society actors play an important role in this process by organizing themselves at a transnational level, engaging with issues that transcend the boundaries of the nation state and questioning the democratic legitimacy of other transnational actors such as international and corporate organizations. They also allow citizens to engage with 'unbounded' issues and to construct a transnational public sphere where such issues can be debated. It is often assumed that the Internet plays a crucial role in enabling this transnational public sphere to take shape. Empirical analysis of discussion forums and mailing lists developed by transnational civil society actors shows, however, that the construction of such a transnational public sphere is paved with constraints. To speak of a unified transnational public sphere is therefore deemed to be problematic. It cannot be seen or construed without taking into account the local, the national, and enforceable rights in order to materialize the ideas and hopes being voiced through civil society."

Coleman, Stephen: The Lonely Citizen: Indirect Representation in an Age of Networks. S. 197-214

Welch, Eric W.: Virtual Interactivity Between Government and Citizens: The Chicago Police Department's Citizen ICAM Application Demonstration Case. - S. 215-236

\section{Public Opinion Quarterly}

\section{Jg 69 (2005) Nr 2}

Price, Vincent: Framing Public Discussion of Gay Civil Unions. - S. 179-212

Ansolabehere, Stephen: Unrepresentative Information: the case of Newspaper Reporting on Campaign Finance. - S. 213-222

Hembroff, Larry A. et al: The Cost-Effectiveness of Alternative Advance Mailings in a Telephone Survey. - S. 232-245

\section{Publizistik}

\section{Jg 50 (2005) Nr 1}

Früh, Werner; Klaus Schönbach: Der dynamisch-transaktionale Ansatz III: eine Zwischenbilanz. - S. 4-20

„Mehr als 20 Jahre nach Erscheinen der beiden Grundlagenartikel zum DTA ergibt eine systematische Recherche der Fachliteratur, dass die Resonanz erstaunlich stark gewesen ist. Der DTA wurde in Lehrbüchern, Theorie- und Forschungsüberblicken beschrieben und diskutiert. Meist geschah dies als eher selbstverständliche Erwähnung, oft aber auch mit positiver oder negativer Wertung. Die hartnäckigsten Einwände, wie Überkomplexität und fehlende empirische Überprüfbarkeit, werden noch einmal aufgegriffen und entkräftet. Insgesamt zeigt sich, dass der DTA nicht nur theoretische Impulse setzte, sondern auch als forschungsleitendes Konzept zu neuen und originellen Erkenntnissen führte."

Donsbach, Wolfgang; Katrin Büttner: Boulevardisierungstrend in deutschen Fernsehnachrichten: Darstellungsmerkmale der Politikberichterstattung vor den Bundestagswahlen 1983, 1990 und 1998. - S. 21-38

„Die Veränderung von Inhalten und Darstellungsformen der Fernsehnachrichten sind ein Indikator dafür, wie der breiten Bevölkerung heute das politische $\mathrm{Ge}$ schehen präsentiert wird. Ein Konzept, mit dem diese Merkmale operationalisiert werden, ist die „Boulevardisierung", das heißt eine Annäherung der Berichterstattung anderer Medien an den Stil von Boulevardblättern und Illustrierten. Um zu ermitteln, ob solche Prozesse auch bei den deutschen Fernsehnachrichten stattgefunden haben, wurde mit einer quantitativen Inhaltsanalyse die Politikberichterstattung in den wichtigsten Nachrichtensendungen von ARD, ZDF, RTL und Sat1 jeweils vier Wochen vor den Bundestagswahlen 1983, 1990 und 1998 untersucht (die Privatsender ab 1990). Grundlage der Analyse bildeten 1241 Beiträge. Tendenzen zu einer stärker boulevardesken Darstellung der Politik sind unverkennbar. Sie zeigen sich auf allen Dimensionen der Boulevardisierung, also in den Inhalten (Rückgang der politischen Beiträge), dem Stil (z. B. steigende Personalisierung) und der Aufmachung (z. B. immer kürzere Sequenzen). Die privaten Sender weisen bei fast allen untersuchten Kriterien erwartungsgemäß stärkere Merkmale der Boulevardisierung auf als die öffentlichrechtlichen Sender. Auffallend stark hat sich aber auch das ZDF diesem Trend angepasst und bei einigen Dimensionen sogar die kommerzielle Konkurrenz überflügelt."

Wünsch, Jana; Bernhard Debatin; Klaus Beck: E-Learning in der Kommunikations- und Medienwissenschaft: Erfahrungen mit dem Einsatz von Online-Lernsystemen in der universitären Lehre. - S. 39-55

„Bei der Nutzung elektronischer Medien in der Lehre handelt es sich nicht um eine Neuheit, aber die rasche Entwicklung und Verbreitung ,neuer Medien wie CD-ROM und WorldWideWeb gaben ihr in den letzten Jahren einen erneuten Auftrieb. So wurden 
auch innerhalb der Kommunikations- und Medienwissenschaft eine Reihe von E-Learning-Projekten entwickelt, über die jedoch bisher ein allgemeiner Überblick fehlte. Der in diesem Artikel vorgestellte Überblick erlaubt eine Einordnung und Bewertung der verschiedenen Projekte und stellt damit auch eine Voraussetzung für den zukünftigen Erfolg solcher Projekte dar. Eine Umfrage der Autoren unter kommunikationswissenschaftlichen Instituten im deutschsprachigen Raum ergab insgesamt 14 E-Learning-Projekte mit zum Teil recht unterschiedlicher inhaltlicher, konzeptioneller, didaktischer, technischer und organisatorischer Ausrichtung. Im Anschluss an die Vorstellung dieser Befragung wird an einer Fallstudie, dem Leipziger ,Distance Learning Propädeutikum (DLP), erläutert und diskutiert, welche Vorteile E-LearningSysteme bieten und welche Schwierigkeiten bei der Entwicklung und dem Einsatz zu meistern sind. Hierzu werden auch Ergebnisse einer projektspezifischen Evaluation herangezogen. Sowohl die Umfrage als auch die Fallstudie zeigen, dass der Hauptvorteil von E-Learning-Systemen nicht so sehr im Ersatz von Lehrveranstaltungen oder der Einsparung von Lehrpersonal liegt, sondern vielmehr in der raum-zeitlichen Flexibilisierung von Lehr- und Lernprozessen.“

Reinemann, Carsten et al: Reliabilität und Validität von RTR-Messungen: ein Vergleich zweier Studien zur zweiten Fernsehdebatte im Bundestagswahlkampf 2002. - S. 56-73

„Dieser Beitrag geht der Frage nach, ob Real-TimeResponse-Messungen valide und reliable Ergebnisse liefern und damit den Anforderungen der empirischen Sozialforschung genügen. Empirisch geprüft wird dies anhand eines Vergleichs zweier in Bamberg und Mainz durchgeführter Quasi-Experimente zum zweiten Fernsehduell im Bundestagswahlkampf 2002. 19 Teilnehmer in Bamberg und 75 Teilnehmer in Mainz verfolgten das zweite TV-Duell auf einer Leinwand. Währenddessen konnten sie - in Echtzeit - ihre Eindrücke von den Kandidaten wiedergeben. Technisch wie inhaltlich unterschieden sich die beiden QuasiExperimente jedoch in wichtigen Details. Dazu zählten die verwendeten RTR-Systeme, die Skalenniveaus der erhobenen Daten und die Anweisungen an die Probanden. Dennoch zeigen die Ergebnisse, dass beide RTR-Messungen reliable Daten liefern, die zudem den Anforderungen an Inhalts-, Konstrukt- und Kriteriumsvalidität entsprechen. Zur Untersuchung subjektiver Eindrücke von Kandidaten in Fernsehdebatten und der längerfristigen Konsequenzen dieser Eindrücke können RTR-Messungen damit einen wertvollen Beitrag leisten."

Neuberger, Christoph: Die Absolventenbefragung als Methode der Lehrevaluation in der Kommunikationswissenschaft: eine Synopse von Studien aus den Jahren 1995 bis 2004. S. $74-103$

„In der Kommunikationswissenschaft sind Befragungen der Absolventinnen und Absolventen eine verbreitete, aber wenig reflektierte Methode der Lehrevaluation. Angesichts des Bedeutungszuwachses der Evaluation an Hochschulen und wegen der großen Konkurrenz auf dem Ausbildungsmarkt scheint es angeraten, sich mit diesem Instrument intensiver zu beschäftigen. Befragungen der Absolventinnen und Absolventen haben den Vorteil, dass diese einen direkten
Vergleich zwischen dem Studium und den in der Berufspraxis gestellten Anforderungen ziehen können. In einer Meta-Analyse wurden 19 Absolventenbefragungen ausgewertet, die in den Jahren 1995 bis 2004 im deutschsprachigen Raum durchgeführt worden sind. Die mangelnde Parallelisierung der Studien erschwert einen solchen Ex-post-Vergleich. Die Ergebnisse zeigen: Der Übergang in den Beruf findet an den Journalistik-Vollstudiengängen besonders rasch statt. Generell ist die Berufserfahrung das wichtigste Einstellungskriterium. Der überwiegende Teil der Befragten würde in den meisten Fällen das Fach wieder wählen. Künftige Absolventenbefragungen sollten koordiniert werden, um Vergleichbarkeit herzustellen und um für das Fach repräsentative Daten zu gewinnen."

\section{Jg 50 (2005) Nr 2}

Reus, Gunter; Lars Harden: Politische „Kultur": Eine Längsschnittanalyse des Zeitungsfeuilletons von 1983 bis 2003. - S. 153-172

„Die Präsentation von Kulturthemen im Feuilleton deutscher Zeitungen hat sich in den vergangenen beiden Jahrzehnten deutlich verändert. Vor allem die ,Politisierung' der Kulturberichterstattung fällt ins Auge. Gleichwohl kann von einem grundlegend neuen Kulturteil keine Rede sein. Das zeigt eine Längsschnittanalyse von vier Tageszeitungen über den Zeitraum von 1983 bis 2003. Das journalistische Angebot im Feuilleton aller vier Zeitungen ist im Zeitverlauf nicht reduziert, sondern kräftig ausgebaut worden. Sowohl die Zahl der Beiträge als auch ihr Umfang haben erheblich zugenommen. Zum wichtigsten Einzelthema ist ,Politik' geworden. Sie hat 2003 einen Anteil an allen Themen von 15 Prozent, verdrängt aber die klassischen Gegenstände Theater, Musik, Bildende Kunst und Literatur keineswegs. Mit einem Themenanteil von rund 50 Prozent bilden sie immer noch den Kernbestand der Kulturberichterstattung. Dabei fällt es dem Ressort auf dem Gebiet der Musik spürbar leichter, sich auch populären und unterhaltungsbetonten Kunstformen zu öffnen, als zum Beispiel auf dem Gebiet des Theaters. Die Rezension ist im Jahre 2003 immer noch unangefochten das häufigste Genre im Feuilleton. Ein ,Ende der Kritik' zugunsten von stark personalisierenden, unterhaltungs- oder servicebetonten Darstellungsformen ist nicht nachweisbar."

Schweiger, Wolfgang: Gibt es einen transmedialen Nutzungsstil?: theoretische Überlegungen und empirische Hinweise. - S. 173-200

„Der Beitrag geht der Frage nach, ob es individuelle Mediennutzungsstile gibt, die sich nicht nur situationsübergreifend, sondern auch medienübergreifend beobachten lassen. Dabei geht es nicht um sog. Medienmenüs, also um die Frage, welche Medienangebote in welchem Maß genutzt werden, sondern um die Art, wie Menschen mit einzelnen Medien umgehen. Wir vermuten etwa, dass Personen, die sich überwiegend spontan für eine TV-Sendung entscheiden, auch bei anderen Mediengattungen ein vergleichbares Auswahlverhalten bevorzugen. Ausgehend von einer theoretischen Einbettung des Konzepts ,transmedialer Nutzungsstil (TMNS) und einigen empirischen Hinweisen wird ein Katalog medienübergreifender Nutzungsstil- Dimensionen mit medienspezifischen 
Operationalisierungsvorschlägen für Fernsehen, Tageszeitung, Zeitschrift und Web erstellt. Dieser Katalog wurde im Sommer 2004 in einer Befragung $n=382$ Personen vorgelegt (repräsentative Quotenstichprobe). Die Ergebnisse bestätigen das Konzept: Fast alle Nutzungsstil-Dimensionen korrelieren auf Individualniveau über Mediengattungen hinweg, die meisten signifikant. Darüber hinaus lässt sich zeigen, wie nahe sich die untersuchten Mediengattungen hinsichtlich ihrer Nutzung aus Rezipientensicht sind. Ein Ausblick diskutiert die Relevanz des Konzepts und skizziert zukünftige Schritte bei der theoretischen und empirischen Beschäftigung mit dem Phänomen."

Meier, Klaus; Frank Feldmeier: Wissenschaftsjournalismus und Wissenschafts-PR im Wandel: eine Studie zu Berufsfeldern, Marktentwicklung und Ausbildung. - S. 201-224

„Das Interesse des Publikums an Wissenschaftsthemen steigt - und gleichzeitig nehmen die medialen Angebote zu: Neue Wissensmagazine und Wissensseiten werden gegründet, Wissenschaftssendungen im Fernsehen eingeführt. Hintergrund und Ursache dieses Trends ist, dass wissenschaftliche Themen zunehmend auf gesellschaftlich zentrale Lebensprozesse einwirken, den Alltag der Menschen betreffen, Emotionen wecken und andere gesellschaftliche Systeme wie Politik, Wirtschaft, Kultur, Religion und Sport beeinflussen. Der Beitrag analysiert diesen gegenwärtigen Wandel von Wissenschaftsjournalismus und Wissenschafts-PR. Auf Basis einer Marktanalyse wurden redaktionelle Führungskräfte aus allen Medienbereichen sowie Leiter von Pressestellen telefonisch befragt. Der Schwerpunkt lag auf Einschätzungen zur Zukunft der Wissenschaftskommunikation, der redaktionellen Verarbeitung von Wissenschaftsthemen und künftigen Qualifikationsanforderungen an Wissenschaftskommunikatoren. Offensichtlich wird der Wissenschaftsjournalismus tagesaktueller, kontroverser und unterhaltsamer. Die Untersuchung legte die Basis für das Konzept eines interdisziplinären Bachelor-Studiengangs, weshalb im Beitrag auch Konsequenzen für die Ausbildung herausgearbeitet werden."

Winterhoff-Spurk, Peter; Frank Schwab: Häufiger, schneller, variabler: Ergebnisse einer Längsschnittuntersuchung über Gewalt in TVNachrichten. - S. 201-224

„Inhaltsanalysen der Hauptnachrichtensendungen deutscher TV-Sender (ARD, ZDF, RTL, SAT.1 und Pro7) aus den jeweils ersten Dezemberwochen der Jahre 1996, 1998, 2000 und 2002 mit rund 40 Stunden Sendematerial und rund 18.000 Einstellungen ergaben: Zahl und Gesamtdauer gewalthaltiger Einstellungen haben zugenommen, die einzelne Einstellung ist kürzer, Einstellungsgröße und Kamerabewegung sind variabler geworden. Von 1996 bis 2000 hatten die privaten Sender hinsichtlich der Zahl und der Gesamtdauer einen rund doppelt so hohen Gewaltanteil wie die öffentlich-rechtlichen. Dieser Unterschied ist im Jahr 2002 auf das Eineinhalbfache gesunken, da nun auch die öffentlich-rechtlichen Sender mehr gewalthaltige Einstellungen zeigten. Die einzelne Einstellung ist bei den privaten Sendern kürzer als bei den öffentlich-rechtlichen, die ,Tagesschau' der ARD ist im Allgemeinen die Nachrichtensendung mit dem geringsten Gewaltanteil und dem vergleichsweise ruhigsten Darbietungstempo.“

\section{Studies in Communication Sciences}

\section{Jg 4 (2004) Nr 2}

Eppler, Martin J.: Information Quality: Organizational, Technological, and Legal Perspectives. - S. $1-16$

"This issue of Studies in Communication Sciences dedicates its thematic section to the topic of information quality and examines the fitness for use of information from three different perspectives: the organizational, technological, and legal viewpoint. We believe that the question of what makes information valuable is essential both in theory and practice, since communication, inter alia, can be understood as the exchange of information aimed at creating and conveying meaning. Exploring the question systematically requires an interdisciplinary approach that highlights the many facets of high quality information, whether they regard content, format, time, process or infrastructure. The interdisciplinary approach, which in our case includes legal scholars, management scientists and information technology researchers, can also help to analyze the plethora of problems related to low quality information, such as misinformation, information overload, paralysis by analysis, wrong decisions, scrap and re-work, or distrust. In this introductory article, we briefly review some of the reasons that make information quality a highly relevant research topic for communication sciences and related disciplines (with numerous practical implications). We offer an overview of the state of the art in information quality research in three disciplines and highlight current research questions as well as unresolved issues. In the last section of this introduction, we provide a synopsis of the various contributions of the thematic section."

Burkert, Herbert: Law and Information Quality: some sceptical observations. - S. 17-28

„Information quality is a subject best to be avoided by law. But it cannot: As primarily an information and communication system, law's procedures, law's products, and law's issues to be decided all painfully evoke questions of information quality: What is it, how is it to be measured, and how can it be ensured? Procedure, product and issue are the three relations of law to information quality I will look at in this contribution. I will call these relations the pragmatic relation (information quality in the context of law's procedures), the syntactic relation (information quality in the context of law's products) and the semantic relation (information quality in the context of issues law has to decide upon). All three relations, I will try to show, are dominated by strategies of avoidance; however, the pressure on law to hold its stand in the last one, the semantic one, is on the increase, but with doubtful success. How doubtful, I will argue, is going to be part of a research program for information law."

Burkert, Herbert: Information Quality and Regulation: a Management Perspective. - S. 2952

"As the impacts of poor quality information affect society and consumers, laws and regulations tend to spring up for protection. While the motivation is to improve information quality and to minimize the im- 
pact of poor information quality, what are the real effects of regulation on information quality? This article is a management analysis, not a legal analysis, exploring some specific examples in the United States of legislation and regulation driven by the impacts of nonquality information on consumers and their impacts and effectiveness on the state of information quality. We then describe fundamental principles of information quality and how they impact information quality, and how a sound quality management system influences the behavior of the organization and the resulting information quality. We describe how a proactive approach to information quality management can mitigate the need for legislation itself and protect the organization from regulatory actions for noncompliance to existing legislation."

\section{Redman, Thomas C.: Barriers to successful} Data Quality Management. - S. 53-68

„As the impacts of poor quality information affect society and consumers, laws and regulations tend to spring up for protection. While the motivation is to improve information quality and to minimize the impact of poor information quality, what are the real effects of regulation on information quality? This article is a management analysis, not a legal analysis, exploring some specific examples in the United States of legislation and regulation driven by the impacts of nonquality information on consumers and their impacts and effectiveness on the state of information quality. We then describe fundamental principles of information quality and how they impact information quality, and how a sound quality management system influences the behavior of the organization and the resulting information quality. We describe how a proactive approach to information quality management can mitigate the need for legislation itself and protect the organization from regulatory actions for noncompliance to existing legislation."

Harpe, Retha de la: An Actor-Network Theory Perspective on Data Quality in Medical Practices. - S. 69-84

„Medical practices in the healthcare sector are today subjected to the same pressures as other businesses. They accumulate large amounts of patient data yet, in most cases, they do not utilize its potential value. Data can only be utilized once its complex nature and flow and the different views and perceptions of all stakeholders are understood. This includes the concept of data quality, which embodies technical and social issues. This paper argues that Actor Network. Theory is a suitable theoretical lens to investigate the actors and their activities in a medical practice in order to improve our understanding of data quality issues. A data network infrastructure is proposed showing the data stakeholders and how they would interact during a consultation. This data network infrastructure can be used to research the complexities of data representation and usage to arrive at a deeper understanding of the data quality issues in medical practices."

Kamthan, Pankaj: A Framework for Addressing the Quality of UML Artifacts. - S. 85-114 „The Unified Modeling Language (UML) is a standard language for modeling the structure and behavior of object-oriented systems. In recent years, there has been a rapid increase in the use of UML artifacts in a variety of application areas. As the use of UML becomes pervasive, the quality of UML artifacts as effective means of communication arises. The aim of this paper is to contribute to a systematic assessment and assurance of the quality of UML artifacts. A quality framework for UML artifacts using notions from semiotics, human-computer interaction, and technical aspects of software diagramming, is proposed. The goals for quality and mechanisms to address them are identified. The mechanisms are themselves analyzed with respect to their usefulness in achieving the quality goals of the artifact. Examples of UML artifacts that compromise quality, and techniques for improvement, are given."

Amicis, Fabrizio de: A Methodology for Data Quality Assessment on Financial Data. S. $115-136$

„This paper proposes a methodology for data quality assessment that has been defined and applied in practice on financial data, in particular for registry data used to describe financial instruments. The methodology encompasses five major phases that define the recognition and classification of primary variables, the data quality analysis techniques and data quality rules used for the inspection of selected data quality dimensions. In the quantitative objective assessment, the measurement of erroneous observations considers the correlation between data quality dimensions. Three independent experts define a qualitative subjective assessment. The results of the two assessments are compared in order to detect discrepancies that are useful for the data quality experts to select actions for data quality improvement. The examples reported in this paper have been selected from a real case."

Cappiello, Cinzia: A Rule-based Methodology to Support Information Quality Assessment and Improvement. - S. 137-154

„Data quality is an increasingly critical issue in the majority of information-intensive businesses. In such contexts, the quality of the information provided is a relevant component for the evaluation of the overall quality of service. A high data quality level is achievable by the adoption of a complete data quality management program that includes algorithms for measuring data quality and automatic techniques for recovering data when their quality decreases below acceptable values. This paper proposes a semi-automatic methodology to perform quality assessment and improvement and to provide support to organizations in achieving a high level of data quality. The methodology takes as input the desired levels of data quality to be obtained and maintained. Evaluation of quality and consequent improvement activities are triggered based on a set of predefined monitoring rules."

Cole, Jonathan: From the Outside In: Towards Shared Perspectives, a Neurological View. S. $155-172$

„The experience of people with neurological impairments, either of facial visible difference or of spinal cord injury, are given in narrative ,neurophenomenological' form. These show how these conditions can alter peoples whole perception of their selfhood and their social interaction. It is suggested that in order to understand these experiences a heightened and deepened form of empathy is required which can also, by extension, be of use in other exchanges and dialogues." 
Bolchini, Davide: Modelling User Requirements for Web Application Design. - S. 173196

„Web applications are designed to communicate for a diversity of purposes to a multiplicity of users with a variety of goals. Analysts have to manage this complexity by modelling user requirements and provide proper guidance to the web design activity. This paper introduces a framework based on user profiles, roles and goals for describing and analysing web user requirements. Project teams can use these conceptual tools for systematically envisioning salient user scenarios at a proper conceptual level. The information gathered through the analysis feeds into a goal-oriented requirements engineering method, which facilitates the transition from requirements to web design. Excerpts from the design of a real museum web site are discussed as a case study."

Lasagni, Christina: European Research on Television and Children: a Survey. - S. 197-228

„This article summarizes a part of a 2003 study codirected by the author analyzing how research in the major European countries, (France, Germany, Italy, the United Kingdom and Spain) has investigated the subject of ,children and television' over the last ten years. With the help of national experts, we selected a sample of 44 pieces of published and unpublished research, mostly carried out between 1990 and 2001 with varying perspectives, objectives and methodologies. The theories, objectives, results and methodologies of this research were analyzed. The study also includes a part dedicated to the European governments' control and regulation of television. The survey focused on many aspects of the relationship between children and television, but here we have selected only a few areas for attention. Many questions, therefore, however interesting, are not dealt with. This includes structural data (how many hours the children spend in front of the television, who they watch it with, at what times of the day, which programs they prefer, etc.). In this article we will also not deal with the topic of the influences exerted by the social context on the use and re-elaboration of television messages, covered in detail in the study. We will instead summarize the parts concerning some particularly relevant questions, for example television violence, or the effects and functions of television from a cognitive point of view. Other topics were selected, because they are new and important in the field of research: for example television's role in sentimental and sexual education and in providing a model of sexual identity. The first part of the article consists of a summary of the most important results to emerge from the 44 studies. The second part consists of some considerations arising from the analysis of the European research about some contents, but also from the evolution of fields of research and methodological tools."

\section{Zeitschrift für Medienpsychologie}

\section{Jg 17 (2005) Nr 2}

Schwonke, Rolf et al: Computergestütztes Schreiben von Lernprotokollen: Umsetzung und Evaluation eines kognitiven Werkzeugs zur Förderung selbstgesteuerten Lernens. S. $42-53$

Petersen, Lars-Eric: Der Einfluss von Models in der Werbung auf das Körperselbstbild der Betrachter/innen. - S. 54-63

Batinic, Bernad: Determinanten der Rücklaufquote in Online-Panels. - S. 64-74

\section{Jg 17 (2005) Nr 3}

Hartmann, Tilo: Ursachen und Effekte parasozialer Interaktionen im Rezeptionsprozess: eine Fragebogenstudie auf der Basis des PSIZwei-Ebenen-Modells. - S. 88-98

„Parasoziale Interaktionen mit Medienfiguren können als interpersonales Involvement von Rezipient/inn/en mit einer Medienperson definiert werden, das sich in perzeptiv-kognitiven, affektiven und konativen Teilprozessen und Erlebensweisen manifestiert. Struktur und Intensität der PSI werden dabei sowohl von den Eigenschaften der Rezipient/inn/en, als auch von den Medienpersonen bzw. ihrer Darstellung beeinflusst. Die vorliegende Studie orientiert sich am PSI-Zwei-Ebenen-Modell nach Hartmann, Schramm und Klimmt (2004a, b) und leitet daraus zwei Ursachen und zwei Effekte von PSIProzessen ab, die für ein personenzentriertes TV-Unterhaltungsformat mittels einer postrezeptiven Befragung geprüft werden. In Übereinstimmung mit den Hypothesen deuten die Ergebnisse darauf hin, dass PSI zu einer Medienfigur umso intensiver ausfallen, je eher diese als attraktiv empfunden wird und je eher sich die Rezipient/inn/en von ihr adressiert fühlen. Intensivere PSI wiederum reduzieren den Grad der Vergegenwärtigung, sich nur in einer medialen Interaktionssituation $\mathrm{zu}$ befinden (Medialitätsbewusstsein), und führen darüber hinaus zu einer positiven Gesamtbewertung des rezipierten Angebots."

Maier, Jens Hendrik: Bestehen parasoziale Beziehungen zu Politikern?: eine empirische Exploration mit der Repertory-Grid-Technik. S. 99-109

„Der Beitrag erschließt die Konzepte PSI/PSB (parasoziale Interaktion/parasoziale Beziehung) für die politische Kommunikationsforschung und erarbeitet eine Möglichkeit, die sozio-emotionalen Dimensionen medialer Politikkommunikation zu untersuchen. Eine kritische Integration der bisherigen Arbeiten zu diesen Konzepten und die Darstellung aktueller politischer Kommunikationspraxis verdeutlichen die Anwendbarkeit des Konzepts PSB. Die Befragung von 118 Proband/inn/en mittels der Repertory Grid Technik zeigt, dass Menschen subjektiv relevante Beziehungen zu Spitzenpolitikern unterhalten und wie diese im Beziehungsraum der Proband/inn/en lokalisiert sind. Die Studie macht einen Methodenvorschlag jenseits der PSI-Skala und überwindet verschiedene Defizite der bisherigen Forschung. So werden auch negativ besetzte Personae untersucht, die Beschränkung auf ein Einzelmedium aufgehoben und die komparative Betrachtung realer und medialer Beziehungen ermöglicht. Die Ergebnisse befürworten eine enge Kooperation zwischen sozialpsychologischer Beziehungsforschung und Medienpsychologie und bringen 
weitere Fortschritte für die jüngeren Bemühungen um die konzeptionelle Weiterentwicklung des Forschungsfelds PSI/PSB.“

Schwab, Frank: MEDIASCOPE: ein System zur Inhalts- und Formalanalyse medialer Angebote. - S. 110-117

„Zur Inhalts- und Formalanalyse audiovisueller Angebote wurde das Auswertungssystem MEDIASCOPE-AV entwickelt. Da audiovisuelle Medien die Realität nicht eins zu eins abbilden, sondern diese narrativ und kameradramaturgisch aufbereiten, werden bei der Analyse mit MEDIASCOPE-AV vor allem die formalen Gestaltungsmerkmale, insbesondere die Kamerahandlung, berücksichtigt. Die formalen Kategorien sind weitgehend genre-übergreifend festgelegt. Die inhaltlichen Kategorien dienen zur Beschreibung der Situation, sie werden je nach Fragestellung und zu analysierendem Genre konkret festgelegt, sind in ihrer Auflösung aber an die formalen Kategorien angepasst. Das System weist insgesamt eine zufrieden stellende Interrater-Reliabilität auf.“

\section{Zeitschrift für Urheber- und Medienrecht}

\section{Jg 49 (2005) Nr 4}

Dreher, Meinrad: Die Beschaffung von Programmmaterial durch Rundfunkanstalten: das kartellvergaberechtliche Umsetzungsdefizit und seine Ausfüllung. - S. 265-274

Koenig, Christian: Ring frei im DVB-T-Beihilfenstreit vor der Europäischen Kommission: terrestrischer digitaler Rundfunk vor dem Aus?. - S. 275-282

„Mit Schreiben vom 14. Juli 2004 hat die Europäische Kommission der Bundesrepublik Deutschland ihren Beschluß mitgeteilt, wegen der finanziellen Förderung des digitalen terrestrischen Rundfunks (Digital Video Broadcasting - Terrestrical, DVB-T) im Großraum Berlin/Potsdam durch die Landesmedienanstalt Berlin-Brandenburg (MABB) ein Beihilfenkontrollverfahren nach Art. 88 II EG einzuleiten. Nachdem die Bundesregierung der Aufforderung der Kommission, eine Stellungnahme in dieser Sache abzugeben, nachgekommen ist, steht die Entscheidung der Kommission bevor. Sollte die Kommission feststellen, daß es sich bei den von der MABB an die private Rundfunkveranstalter ausgekehrten Fördermitteln um gemeinschaftsrechtwidrige Beihilfen handelt, würde dies einen empfindlichen Rückschlag für die geplante bundesweite Einführung des digitalen terrestrischen Fernsehens bedeuten."

Castendyk, Oliver: „Man spricht deutsh“ zwischen den Instanzen: zum Verhältnis von nationalen und europäischen Urheberrecht am Beispiel des $₫ 137$ h Abs. 2 UrhG; Besprechung von BGH ZUM 2005, 69ff. - S. 283-288

König, Michael: Die digitale Zugangsfreiheit im 8. RÄStV: nur Anpassung an das TKG oder materielle Änderungen beabsichtigt?. - S. 289297
Die Ministerpräsidenten der Länder haben am 8. Oktober 2004 den 8. Rundfunkänderungsstaatsvertrag (RÄStV) unterzeichnet. Im Schatten der viel diskutierten Erhöhung der Rundfunkgebühr wurde eher unbemerkt auch eine Neufassung von $\mathbb{5} 33$ Rundfunkstaatsvertrag (RStV) vorgenommen. Laut Begründung soll diese Neufassung der Anpassung des Medienrechts der Länder an das neue Telekommunikationsgesetz (TKG) dienen. Zusätzlich ist mit der Neufassung des $\ 53 \mathrm{RStV}$ lediglich eine Komprimierung und Verallgemeinerung der bisherigen Norm beabsichtigt. Der neue Wortlaut lässt allerdings in einigen Punkten unterschiedliche und von der alten Regelung erheblich abweichende Auslegungen zu. Dieser Beitrag will unter Berücksichtigung der bisher geltenden Fassung von $\$ 53 \mathrm{RStV}$ und der entsprechenden TKG-Regelung die kritischen Punkte der neuen Fassung aufzeigen und hinsichtlich des Kreises der Adressaten und Berechtigten der Norm näher betrachten. Außerdem wird untersucht, inwiefern die Neuregelung eine Entgeltregulierung durch die Landesmedienanstalten vorsieht. Überprüft wird in diesem Zusammenhang auch, ob die geänderte Fassung eine praktikablere Abgrenzung der Kompetenzen der Regulierungsbehörde für Telekommunikation und Post (Reg TP) und der Landesmedienanstalten vorsieht. Im Zentrum der Untersuchung stehen jedoch die Regelungen zu Zugangsberechtigungssystemen („Conditional Access“).

Kitz, Volker: $\$ 101$ a UrhG: Für eine Rückkehr zur Dogmatik. - S. 298-302

Becker, Bernhard von: „Juristisches Neuland“: angemessene Vergütung, Vergütungsregeln, Zweckübertragungsregel. - S. 303-306

Reinemann, Susanne: Rundfunkvermarktung und Informationsfreiheit: Tagungsbericht über das 7. BLM-Symposion Medienrecht in München. - S. 307-335

Ernst, Stefan: Internet-Suchmaschine: Anmerkung zu LG Hamburg, Urt. v. 21.9.2004. S. 336-340

\section{Jg 49 (2005) Nr 5}

Gundel, Jörg: Das Verbot der ideellen Werbung auf dem Prüfstand der EMRK. - S. 345-352

Dieser Beitrag beschäftigt sich mit der Frage der Zulässigkeit ideeller Rundfunkwerbung, also der Verbreitung nichtkommerzieller, sondern politischer oder religiöser Werbebotschaften im Rahmen der üblicherweise der Wirtschaftswerbung gewidmeten Werbeunterbrechungen im Rundfunkprogramm. Der EGMR hat in zwei jüngeren Entscheidungen die Vereinbarkeit eines Verbots solcher Werbebotschaften im Rundfunk mit Art. 10 EMRK geprüft. Er ist dabei zu uneinheitlichen Ergebnissen gekommen, die auch für die bestehende Verbotsregelung des deutschen Rundfunkrechts Fragen aufwerfen.

Beuthien, Volker: Bildberichte über aktive und passive Personen der Zeitgeschichte: unterschiedliche Persönlichkeitsschutzgrenzen. - S. 352-356 
Das in $\$ 23$ KUG als besonderes Persönlichkeitsrecht gesetzlich vorausgesetzte Recht am eigenen Bilde hat sich in der Mediengesellschaft zu einem wesentlichen personalen Schutzgut entwickelt. Entsprechend gestiegen ist aber auch das Interesse der Öffentlichkeit, über das Leben und Wirken prominenter Personen bildlich oder bildgestützt unterrichtet $\mathrm{zu}$ werden. Deshalb kommt es mehr denn je darauf an, wer „Person der Zeitgeschichte“ im Sinne des \$2 3 I Nr. 1 KUG ist und sich daher, falls er nicht gem. \ 23 II KUG berechtigte Gegeninteressen geltend machen kann, ohne Einwilligung ablichten lassen muss. Vor diesem Hintergrund beschäftigt sich vorliegender Beitrag mit dem Begriff der Zeitgeschichte und den berechtigten Gegeninteressen i. S. d. \$ 23 II KUG. Abschließend werden daraus Folgerungen für die Caroline-Rechtsprechung gezogen.

Emmer, Thomas: Steht das Vermietrecht des Urhebers der Erhebung eines Büchergeldes entgegen?. - S. 356-359

Gottschalk, Eckart: Wettbewerbsverbote in Verlagsverträgen. - S. 359-368

Abdallah, Tarek: Strafrechtliche und strafprozessuale Probleme der Ermittlung nutzerbezogener Daten im Internet: dargestellt am Beispiel des Tauschs urheberrechtlich geschützter Werke in Filesharing-Netzwerken. - S. 368-376

Hartz, Nikolaus v.: Beweissicherungsmöglichkeiten im Urheberrecht nach der EnforcementRichtlinie im deutschen Recht. - S. 376-410

\section{Jg 49 (2005) Nr 6}

Sauren, Jürgen: Bedrohung der freien Berichterstattung durch den neuen $\int 201$ a StGB?. S. 425-432

Mit dem 36. Strafrechtsänderungsgesetz ist am 6. August 2004 der $\$ 201$ a StGB in Kraft getreten. Das Gesetzgebungsverfahren wurde jedoch durch eine beträchtliche Anzahl kritischer Stimmen aus dem Bereich der juristischen Literatur sowie aus dem Bereich der Medienvertreter begleitet, da eine Bedrohung der freien Berichterstattung befürchtet wurde. Seit einigen Monaten ist die Norm nun Realität und die Frage nach der Bedrohung der freien Berichterstattung stellt sich konkret. Mit diesem Beitrag möchte der Autor, nach einem Überblick über die Entstehung des Gesetzes, den Inhalt der Norm erörtern und eigene kritische Bedenken äußern.

Hesse, Albrecht: Der $\$ 201$ a StGB aus Sicht des öffentlich-rechtlichen Rundfunks. - S. 4342436

Am 6. August 2004 ist $\$ 201$ a StGB (Verletzung des höchstpersönlichen Lebensbereichs durch Bildaufnahmen) in Kraft getreten, der unter bestimmten Voraussetzung bereits die Anfertigung von Bildnissen unter Strafe stellt. Damit ist die Schwelle für Eingriffe in Persönlichkeitsrechte erheblich nach vorne verlagert und dementsprechend sind der Bildberichterstattung engere Grenzen gezogen worden. Dies bringt für den öffentlichen Rundfunk eine Reihe von Problemen mit sich, die in diesem Beitrag aufgegriffen und bespro- chen werden: Da es sich bei $\ 201$ a StGB um einen Straftatbestand handelt, kann eine Verletzung dieser Vorschrift eine Strafverfolgung nach sich ziehen. Dies trifft insbesondere den an der Kamera tätigen Mitarbeiter, aber u. U. auch den Redakteur persönlich. Die Rundfunkanstalt kann ihnen dieses Risiko jedoch nicht abnehmen. Zudem muß die Kontrolle der rechtlichen Zulässigkeit bereits vor der Aufnahme erfolgen. Hierfür stehen $u$. U. allerdings nur wenige Augenblicke zur Verfügung. Ein weiteres Risiko besteht darin, daß bei strafrechtlichen Ermittlungsverfahren Durchsuchungen und Beschlagnahme drohen können, mit negativen Folgen für die Bereitschaft potenzieller Informanten, der Presse oder dem Rundfunk brisante Informationen zukommen zu lassen. Im Ergebnis führt die Vorschrift zu einer Verunsicherung der im Bereich des Rundfunks tätigen Mitarbeiter.

Obert, Anne: Der $\$ 201$ a StGB aus Sicht des privaten Rundfunks. - S. 436-441

Der deutsche Gesetzgeber hat sich im vergangenen Jahr trotz erheblicher und einvernehmlicher Kritik der Medienverbände und -unternehmen entschieden, Persönlichkeitsrechtsverletzungen strafrechtlich zu sanktionieren. Der neue $\ 201$ a StGB stellt u. a. bereits das Herstellen von Bildaufnahmen unter Strafe, unabhängig von einer etwaigen nachfolgenden Veröffentlichung. Er verfolgt das Ziel, den höchstpersönlichen Lebensbereich des Einzelnen vor unbefugten Bildaufnahmen zu schützen. Die praktische Relevanz dieser Vorschrift betrifft nicht nur Nachrichtensendungen und Boulevardmagazine, sondern in erster Linie investigative Formate - insbesondere, wenn eine versteckte Kamera eingesetzt wird. In diesem Beitrag wird zunächst die wissenschaftliche Diskussion kritisch gewürdigt. Anschließend wird auf die verschiedenen Tathandlungen des $\$ 201$ a StGB und auf die Rechtfertigungsmöglichkeiten eingegangen. Ziel dieses Beitrages ist es, die neue Strafvorschrift aus Sicht des privaten Rundfunks darzustellen.

Tillmanns, Lutz: Der $\$ 201$ a StGB: eine problemorientierte Betrachtung aus Sicht der Presseselbstregulierung. - S. 441-449

Seit August 2004 können sich Paparazzi und seriöse Fotografen aufgrund des neuen \$201 a StGB leichter strafbar machen. Stellvertretend für viele mahnten jüngst Journalisten auf einer Podiumsdiskussion zum Thema „Prominentenanwälte contra Pressefreiheit" die schwieriger gewordenen Bedingungen für die Arbeit der Presse an, die nicht zuletzt durch die aktuellen Entwicklungen in Rechtsprechung und Gesetzgebung hervorgerufen wurden. Es sei mehr denn je erforderlich, die Pressearbeit juristisch begleiten zu lassen, um die Grenzen des Rechts einhalten zu können. Dieser Umstand erhöhe nicht unbedingt die Qualität der Pressearbeit, die in nicht unerheblichem Maß von der Aktualität der Informationen abhängt. Vor diesem Hintergrund beschäftigt sich dieser Beitrag mit den praktischen Problemen der journalistischen Arbeit, der Erforderlichkeit dieser neuen Regelung, der Unbestimmtheit einzelner Tatbestandsmerkmale von \201 a StGB und gibt zum Abschluss eine rechtpolitische Abschätzung mit Ausblick.

Vogel, Simon: Bedrohung der freien Berichterstattung durch den neuen $\mathbb{S} 210 \mathrm{a}$ StGB?: Gleichnamige Sitzung des Instituts für Ur- 
heber- und Medienrecht vom 11. März 2005. S. $449-452$

Ullrich, Michael: Die Bewertung von Rundfunkprogrammen durch Einrichtungen der Freiwilligen Selbstkontrolle und ihre Folgen. S. $452-462$

Am 1. April 2003 ist der Staatsvertrag über den Schutz der Menschenwürde und den Jugendschutz in Rundfunk und Telemedien (JMStV) in Kraft getreten. Das erklärte Ziel dieses Vertrages war gem. \1 $1 \mathrm{JMStV}$ die Vereinheitlichung der Schutzstandards für Minderjährige vor bestimmten Angeboten in elektronischen Informations- und Kommunikationsmedien, da bislang - abhängig vom Medium - recht unterschiedliche Anforderungen galten. Ein weiterer Schwerpunkt lag in der Neugestaltung der Aufsicht im Bereich des privaten Rundfunks und der Telemedien. Diese obliegt gem. \14 I JMStV nach wie vor grundsätzlich der zuständigen Landesmedienanstalt. Daneben läßt \19 I JMStV jedoch auch die Bildung von Einrichtungen der freiwilligen Selbstkontrolle im Bereich des Rundfunks und der Telemedien zu. Dieses dualistisch strukturierte System der Medienaufsicht birgt ein erhebliches Konfliktpotential, da hier die Verantwortungsbereiche von staatlicher Medienaufsicht und Freiwilliger Selbstkontrolle aufeinander treffen. In diesem Beitrag wird die Arbeit von Einrichtungen der Freiwilligen Selbstkontrolle bei der Bewertung von Rundfunkprogrammen am Beispiel der Freiwilligen Selbstkontrolle Fernsehen e. V. (FSF) untersucht, um so Rückschlüsse auf die rechtliche Stellung dieser Einrichtungen und ihr Verhältnis zur staatlichen Aufsicht ziehen zu können.
Cole, Mark D.: Der Dualismus von Selbstkontrolle und Aufsicht im Jugendmedienschutz: zum Verhältnis von FSF und KJM im System der „regulierten Selbstregulierung“: eine Untersuchung aus Anlass des Konflikts um Schönheitsoperationen im Fernsehprogramm. S. $462-482$

Vor zwei Jahren wurde der Jugendschutz - nicht zuletzt unter dem Eindruck des Erfurter Amoklaufs grundlegend reformiert. Damals wurde neben einer Präzisierung der materiellen Jugendschutzbestimmungen als wesentliche Änderung eine Vereinheitlichung der Jugendschutzvorschriften für elektronische Medien voran gebracht und zudem das System der freiwilligen Selbstkontrolle der Anbieter gestärkt. Dabei wurde eine neue Kommission für Jugendschutz (KJM) als zentrale Kommission der Landesmedienanstalten eingerichtet, die für die wesentlichen Aufsichtsbefugnisse beim Jugendschutz bzgl. der elektronischen Medien zuständig ist. $\mathrm{Zu}$ ihren Aufgaben gehört unter anderem die Anerkennung von freiwilligen Selbstkontrolleinrichtungen und damit eine ganz neue Form eines sozusagen ,staatsfern erteilten Quasi-TÜVs". Zwei Jahre nach dieser grundlegenden Reform ist die Zeit reif für eine erste Bilanz über das Funktionieren der wesentlichen Änderungen. Diese Bewertung in vorliegendem Beitrag erfolgt nicht nur aus grundsätzlichen Erwägungen heraus, sondern auch weil sich zwischenzeitlich im Konflikt über die Ausstrahlung von Fernseh-Shows rund um Schönheitsoperationen konkrete Fragen zum Verhältnis zwischen den Selbstkontrolleinrichtungen und der neu geschaffenen KJM ergeben. 